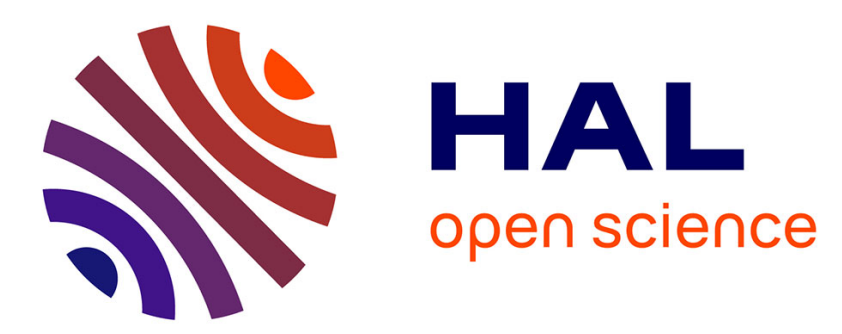

\title{
Numerical modeling of elasto-plastic porous materials with void shape effects at finite deformations
}

\author{
Kostas Danas, N. Aravas
}

\section{To cite this version:}

Kostas Danas, N. Aravas. Numerical modeling of elasto-plastic porous materials with void shape effects at finite deformations. Composites Part B: Engineering, 2012, 43, pp.2544-2559. 10.1016/j.compositesb.2011.12.011 . hal-00755844

HAL Id: hal-00755844

https://hal-polytechnique.archives-ouvertes.fr/hal-00755844

Submitted on 21 Dec 2017

HAL is a multi-disciplinary open access archive for the deposit and dissemination of scientific research documents, whether they are published or not. The documents may come from teaching and research institutions in France or abroad, or from public or private research centers.
L'archive ouverte pluridisciplinaire HAL, est destinée au dépôt et à la diffusion de documents scientifiques de niveau recherche, publiés ou non, émanant des établissements d'enseignement et de recherche français ou étrangers, des laboratoires publics ou privés. 


\title{
Numerical modeling of elasto-plastic porous materials with void shape effects at finite deformations
}

\author{
K. Danas ${ }^{\mathrm{a}}, \mathrm{N}$. Aravas $^{\mathrm{b}, \mathrm{c}, *}$ \\ ${ }^{a}$ Laboratoire de Mécanique des Solides, C.N.R.S. UMR7649, École Polytechnique, ParisTech, 91128 Palaiseau Cedex, France \\ ${ }^{b}$ Department of Mechanical Engineering, University of Thessaly, Pedion Areos, 38334 Volos, Greece \\ ${ }^{c}$ The Mechatronics Institute, Center for Research and Technology - Thessaly (CERETETH), 1st Industrial Area, 38500 \\ Volos, Greece
}

\begin{abstract}
A new constitutive model for elasto-plastic (rate-independent) porous materials subjected to general three-dimensional finite deformations is presented. The new model results from simple modifications of an earlier model of Kailasam and Ponte Castañeda $(1997,1998)$ so that it reproduces the exact spherical and cylindrical shell solution (composite shere and composite cylinder assemblage) under purely hydrostatic loadings, while predicting (by calibration) accurately the void shape evolution according to the recent "second-order" model of Danas and Ponte Castañeda (2009a). Furthermore, the present model is based on a rigorous homogenization method which is capable of predicting both the constitutive behavior and the microstructure evolution of porous materials. The microstructure is described by voids of arbitrary ellipsoidal shapes and orientations and as a result the material exhibits deformation-induced (or morphological) anisotropy at finite deformations. This is in contrast with the well-known Gurson (1977) model which assumes that the voids remain spherical during the deformation process and thus the material remains always isotropic. The present model is implemented numerically in a finite element program where a three-dimensional thin-sheet (butterfly) specimen is subjected to a combination of shear and traction loading conditions in order to examine the effect of stress triaxiality and shearing upon material failure. The ability of the present model to take into account the nontrivial evolution of the microstructure and especially void shape effects leads to the prediction of material failure even at low stress triaxialities and small porosities without the use of additional phenomenological damage criteria. At high stress triaxialities, the present model gives similar predictions as the Gurson model.
\end{abstract}

Key words: C. Computational modelling, C. Finite element analysis (FEA), B. Anisotropy, Homogenization

\section{Introduction}

A critical mechanism of ductile failure in metals is more often than not the nucleation, growth and eventual coalescence of voids and micro-cracks as a result of the applied loading conditions (Johnson and Cook, 1985; Garrison and Moody, 1987). On the other hand, it is worth mentioning a few exceptions (Ghahremaninezhad and Ravi-Chandar, 2011) such as extremely pure materials where fracture occurs due to the extreme shearing and subsequent rupture of grains as a result of high shear strains and dislocation saturation. In general, however, the presence of pores (i.e., porosity) is an undesirable and uncontrollable fact that could be due to pre-existing porosity in the metallic (matrix) phase or nucleation of pores in the neighborhood of impurities which are brittle and thus tend to fracture even at small strains.

\footnotetext{
* Corresponding author.

Email addresses: kdanas@lms.polytechnique.fr (K. Danas), aravas@uth.gr (N. Aravas)
} 
It has been known for many years that the stress triaxiality, denoted here by $X_{\Sigma}$ and defined as the ratio of the mean stress to the von Mises equivalent or effective deviatoric stress, is at least one of the critical parameters controlling ductile failure especially at high triaxialities. This is a direct consequence of the significant growth rate of the voids at large triaxialities (due to high tensile hydrostatic stresses), which lead to the coalescence of neighboring voids and final fracture of the solid.

Nonetheless, recent experiments (Bao and Wierzbicki, 2004; Barsoum and Faleskog, 2007a; Mohr and Ebnoether, 2009; Dunand and Mohr, 2010, 2011) suggest that a new, different mechanism should come into play at low triaxialities. Indeed, in these studies, it has been found that a second loading parameter, the Lode parameter, $L$ (or equivalently Lode angle, $\theta$ ) also plays a significant role in ductile failure at low triaxialities. The Lode parameter is a function of the third invariant of the stress deviator and is used to distinguish between the different shear stress states in three dimensions (3-D). At low triaxialities, careful experimental observations (Benzerga et al., 2004a; Barsoum and Faleskog, 2007a; Boisot et al., 2011) strongly suggest that, void elongation and rotation, which is dependent on the specific shear stress state and leads to a deformation-induced morphological anisotropy during the deformation process, becomes the dominant mechanism leading to the failure of the material. Therefore, modeling of material failure should also take into account void shape effects.

Modeling of the aforementioned mechanisms, such as void growth and change of void shape and void orientation, has been the subject of numerous studies in the literature over the last forty years. The wellknown Gurson (1977) model (and its modifications by Tvergaard (1981)) has been the first complete and analytical model which is based on a micromechanical analysis of a spherical shell (based on an earlier work of Rice and Tracey (1969)), assumed to remain spherical even for general loading conditions (i.e., the solid remains isotropic even at large shearing strains). Even though this assumption is entirely consistent with the void growth mechanisms observed under pure hydrostatic stress states, it becomes less adequate with the addition of shear loads, since such loads can induce significant morphological anisotropy due to changes in void shapes. In an attempt to circumvent this drawback, several modifications of the original Gurson model have been proposed in later studies. Specifically, making use of a confocal spheroidal shell, Gologanu et al. $(1993,1994,1997)$ proposed a model for porous materials with aligned spheroidal voids that are subjected to axisymmetric stress states aligned with the voids symmetry axis (see also later works by Gărăjeu et al. (2000), Benzerga (2002), Benzerga et al. (2004b), Flandi and Leblond (2005a), Monchiet et al. (2007), Vincent and Monerie (2008) for random distribution of cracks and the review article Benzerga and Leblond (2010) for a complete set of references). Nonetheless, spheroidal void shapes are a consequence of axisymmetric loading conditions that are aligned with the symmetry axis of the void. Thus, these models are unable to handle more general loading conditions including general void shape changes and void rotation under non-aligned loadings and finite deformations (but see Leblond and Gologanu (2008) who have reported work in progress along these lines albeit without considering the underlying evolution of microstructure).

In contrast to these micromechanical single-shell approaches, more general constitutive models for porous ductile materials capable of accounting for pore shape and orientation evolution-under general threedimensional loading conditions - have been developed by Ponte Castañeda and Zaidman (1994), Kailasam et al. (1997a,b) and Kailasam and Ponte Castañeda (1997) for porous elasto-plastic materials. These models make use of the "variational" (VAR) linear comparison homogenization method of Ponte Castañeda (1991a) (see also Willis (1991), Michel and Suquet (1992), Suquet (1993) and Suquet (1995) who interpreted the variational method as a "secant" method), together with the estimates of Ponte Castañeda and Willis (1995) for porous linear-elastic materials with "ellipsoidal" microstructures (i.e., particulate microstructures containing orthotropic distributions of ellipsoidal pores), to generate corresponding estimates for the macroscopic response of elasto-plastic porous materials. They are supplemented by evolution laws for microstructural variables corresponding to the porosity, average pore shape and orientation, which are obtained from the homogenization analyses in a self-consistent fashion (Ponte Castañeda and Zaidman, 1994; Kailasam and Ponte Castañeda, 1998). The variational non-linear homogenization method has also been extended to include strain hardening elasto-plastic behavior for the matrix material, and implemented numerically in large-scale, structural finite element programs by Kailasam et al. (2000) and Aravas and Ponte Castañeda (2004).

While these models are quite general, they tend to give overly stiff predictions at high triaxialities and 
small porosities. However, this limitation has been removed, at least for isotropic matrix systems, in recent work by Danas and Ponte Castañeda (2009a,b), making use of the more accurate "second-order" linear comparison homogenization method of Ponte Castañeda (2002a,b), and building on earlier works by Danas et al. (2008a), Danas et al. (2008b) and Danas (2008). The resulting model, which will be referred to here as the SOM model, is also capable of handling general ellipsoidal particulate microstructures and general threedimensional loading conditions, including those leading to pore rotation, while remaining quite accurate at large stress triaxialities and recovering the Gurson model for purely hydrostatic loadings and spherical or cylindrical pores. In a recent work by Danas and Ponte Castañeda (under review), where the SOM model has been extended to account for elasto-plastic behavior with strain-hardening of the matrix phase, it has been shown that it is able to predict material failure even at very low positive stress triaxialities as a result of a extreme void elongation that leads to void collapse and loss of the loading carrying capacity of the solid (see also Tvergaard (2009) in two-dimensions).

In view of the above described methods, it is useful to identify a few major differences between the Gurson-type micromechanical models and the nonlinear homogenization methods. A major strength of the nonlinear homogenization models lies in the fact that they describe representative volume elements and thus take into account - albeit in an approximate manner through at most two-point correlation functions — void-void interactions (Idiart et al., 2006), which can become significant at strong nonlinearities (e.g., plasticity), even at tiny volume fractions. On the contrary, the micromechanical models are based on an approximate limit analysis of a single spherical or confocal spheroidal shell subjected often to uniform strain boundary conditions. This obviously neglects any void interactions of neighboring voids in the solid except in very distinct cases that the single shell model can be identified with a composite material. Such cases are the composite sphere or cylinder assemblage of Hashin (1962) which is valid only for purely hydrostatic loadings and the "confocal spheroids" of Gologanu et al. (1993, 1997) which are valid only for a unique axisymmetric loading which depends on the shape of the spheroid (in the limit that the spheroid becomes a sphere one recovers the composite sphere assemblage and the relevant loading is purely hydrostatic). An additional, but not least, advantage of the homogenization methods over the micromechanical single-shell models is related to the fact that they can be used to generate consistent evolution laws for the relevant microstructural variables contrary to the micromechanical models such as Gologanu et al. (1997), which need to borrow evolution laws for the pore orientation from other models (e.g., above-mentioned homogenization models and/or unit cell finite element models).

\subsection{Scope of the study}

The scope of the present study is twofold. First, we propose the modified variational model (MVAR) for elasto-plastic, rate-independent porous materials. This model is obtained by simple modifications of the earlier "variational" homogenization method which is known to be stiff at high stress triaxialities but is accurate at low stress triaxialities, while it also tends to underestimate the evolution of the void shapes. These modifications allow for very accurate predictions of the macroscopic response and microstructure evolution of porous materials subjected to general three-dimensional loading conditions at both low and high stress triaxialities, as is the case in the SOM model of Danas and Ponte Castañeda (2009a). Comparison of the MVAR prediction with the SOM and Gurson estimates will be carried out for both the instantaneous response (i.e., yield surfaces) and the microstructure evolution (i.e., stress-strain curves).

Second, the new MVAR model is implemented numerically in a three dimensional user-material subroutine (UMAT) in the Abaqus (2009) general-purpose finite element code by proper modification of the earlier two-dimensional version developed by Kailasam et al. (2000) and Aravas and Ponte Castañeda (2004). The MVAR model, even though being less accurate than the SOM model at the level of the representative volume element, it is more stable and faster numerically than the SOM model since it involves only the evaluation of one-dimensional elliptic integrals of the Eshelby-type, contrary to the SOM model that requires, in the more general case, the numerical evaluation of two-dimensional (surface) integrals which become singular at large void elongations. For completeness, the MVAR model will also be compared with the original Gurson (1977) model which is also implemented numerically in a user-material subroutine.

Following the introduction, we describe the microstructure making use of the appropriate microstructural variables. Then the theory is presented in two separate sections. The first section discusses the modification 
of the original variational method (i.e., MVAR model) to correct for the material response at high stress triaxialities and makes contact with the composite sphere and composite cylinder assemblages of Hashin (1962). The second section discusses the evolution equations for the microstructural variables used in the present model. The theory is followed by two sections where the MVAR model is compared with the SOM and the Gurson models in the prediction of the yield surfaces and the microstructure evolution of the porous material. Then, numerical simulations of a three-dimensional thin-sheet (butterfly) specimen are carried out using the MVAR and the Gurson models and the corresponding results of the two models are compared for shear-dominated and traction loading conditions.

\section{Microstructure}

The porous material is composed of two phases. The matrix phase is elasto-plastic (rate-independent) and isotropic following a $J_{2}$ flow rule with strain hardening described by the yield stress $\sigma_{y}$ as a function of the accumulated equivalent plastic strain $\varepsilon_{M}^{p}$. The inclusion phase is vacuous and comprises voids of the same shape and orientation distributed uniformly over the representative volume element. As a consequence of the finite deformations considered in this work, the voids evolve into ellipsoidal shapes and hence the porous medium becomes locally anisotropic (i.e., develops morphological anisotropy). Consequently, it is necessary to define microstructural variables that not only describe the volume fraction of the voids, as is the case in the models of Gurson (1977) and Nahshon and Hutchinson (2008), but also their shape, distribution and orientation.

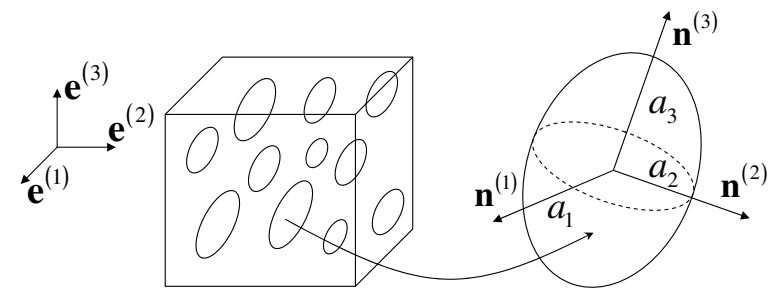

Figure 1: Graphical representation of the microstructure showing the local orientation axes $\mathbf{n}^{(i)}$ with $i=1,2,3$ of a representative ellipsoidal void with semi-axis $a_{1}, a_{2}$ and $a_{3}$.

According to the schematic representation shown in Fig. 1 and at some finite deformation state, we consider that the porous material is characterized by a "particulate" microstructure consisting of ellipsoidal voids with semi-axes $a_{1} \neq a_{2} \neq a_{3}$ aligned in a certain direction. In addition, it is assumed (Willis, 1978, 1981; Ponte Castañeda and Willis, 1995) that the centers of the voids are distributed with ellipsoidal symmetry, i.e., the distribution (or two-point correlation) function of the centers of the voids has also ellipsoidal shape. This description of a particulate microstructure represents a generalization of the Eshelby (1957) dilute microstructure to the non-dilute regime. In this work, which is based in the models of Ponte Castañeda and Zaidman (1994) and Danas and Ponte Castañeda (2009a), we will make the simplifying assumption that the ellipsoidal shape and orientation of the distribution function is identical to the ellipsoidal shape and orientation of the voids at each stage of the deformation. This assumption has been shown (Danas and Ponte Castañeda, 2009b) to provide very accurate estimates, especially at small and moderate porosities. Nonetheless, it should be mentioned that, in general, the void distribution shape could be different from the void shape, as discussed by Ponte Castañeda and Willis (1995), and this effect could be accounted for at least approximately (Kailasam et al., 1997a).

In view of the above hypotheses, the relevant internal variables describing the state of the microstructure in this problem are:

$$
s_{\alpha}=\left\{\varepsilon_{M}^{p}, f, w_{1}, w_{2}, \mathbf{n}^{(1)}, \mathbf{n}^{(2)}, \mathbf{n}^{(3)}=\mathbf{n}^{(1)} \times \mathbf{n}^{(2)}\right\},
$$

where $\varepsilon_{M}^{p}$ is the accumulated plastic strain in the undamaged matrix phase, $f$ is the porosity (i.e., volume fraction of the voids), and $w_{1}=a_{3} / a_{1}$ and $w_{2}=a_{3} / a_{2}$ are two aspect ratios characterizing the ellipsoidal shape of the voids (with $a_{1}, a_{2}$ and $a_{3}$ denoting the principal semi-axes of the ellipsoidal voids) and their 
distribution function, while the vectors $\mathbf{n}^{(i)}$ (with $i=1,2,3$ ) denote the orientation of the principal axes of the voids.

\section{Instantaneous constitutive relations}

The overall strain-rate $D_{i j}=\left(v_{i, j}+v_{j, i}\right) / 2$ (with $v_{i}$ denoting the cartesian components of the overall applied velocity) is decomposed as

$$
D_{i j}=D_{i j}^{e}+D_{i j}^{p},
$$

where $D_{i j}^{e}$ and $D_{i j}^{p}$, respectively, denote the elastic and plastic parts. Note that due to the presence of voids the overall material behavior is compressible implying that the plastic strain-rate tensor is not deviatoric (i.e., $D_{k k}^{p} \neq 0$ ). In view of the fact that the pores can carry no loads and following Aravas and Ponte Castañeda (2004), it is assumed that the elastic and plastic parts of the strain rate can be estimated by independent, but consistent homogenization analyses.

\subsection{Elasticity}

Thus, the elastic response of the porous material is described in terms of an effective compliance tensor M via

$$
D_{i j}^{e}=M_{i j k l} \stackrel{\nabla_{\sigma}}{\sigma k l}, \quad M_{i j k l}=M_{i j k l}^{M}+\frac{f}{1-f} Q_{i j k l}^{-1},
$$

where $\stackrel{\nabla}{\sigma}$ is the Jaumann rate of the Cauchy stress, i.e.,

$$
\stackrel{\nabla}{\sigma}_{i j}=\dot{\sigma}_{i j}-W_{i k} \sigma_{k j}+\sigma_{i m} W_{m j},
$$

with $W_{i j}=\left(v_{i, j}-v_{j, i}\right) / 2$ denoting the overall applied macroscopic spin.

In relation (3), the microstructural fourth-order tensor, $Q_{i j k l}$, is directly related to the well-known Eshelby (1957) or Hill (1978) tensor for ellipsoidal microstructures and its evaluation is detailed in the Appendix of Aravas and Ponte Castañeda (2004). The fourth-order tensor $\mathbf{M}^{M}$ is the compliance modulus of the matrix phase and is taken to be isotropic such that

$$
M_{i j k l}^{M}=\frac{1}{2 \mu} K_{i j k l}+\frac{1}{3 \kappa} J_{i j k l},
$$

where $\mu$ and $\kappa$ denote the elastic shear and bulk moduli of the matrix phase, respectively, with

$$
I_{i j k l}=\frac{1}{2}\left(\delta_{i k} \delta_{j l}+\delta_{i l} \delta_{j k}\right), \quad J_{i j k l}=\frac{1}{3} \delta_{i j} \delta_{k l}, \quad K_{i j k l}=I_{i j k l}-J_{i j k l} .
$$

In this last expression, $I_{i j k l}, J_{i j k l}$ and $K_{i j k l}$ denote the cartesian components of the standard, fourth-order, identity, hydrostatic and shear projection tensors, respectively.

\subsection{Plasticity}

The present study focuses on rate-independent, elasto-plastic porous materials and subsequent results include no rate effects. However, for the derivation of the rate-independent constitutive relations for the porous material, it is useful to start by considering the appropriate limit of a homogenized dissipation (rate-dependent) potential $U\left(\sigma_{i j}\right)$ proposed by Ponte Castañeda (1991a) (but see for more details regarding porous materials the doctoral thesis of Danas (2008)), which takes the form

$$
U\left(\boldsymbol{\sigma} ; s_{\alpha}\right)=(1-f) \frac{\dot{\varepsilon}_{o} \sigma_{y}}{n+1}\left[\frac{\widehat{\sigma}_{e}}{\sigma_{y}\left(\varepsilon_{M}^{p}\right)}\right]^{1+n} .
$$

Here, $\widehat{\sigma}_{e}\left(\boldsymbol{\sigma} ; s_{\alpha}\right)$ contains all the information about the microstructure and is detailed later in this section, $\sigma_{y}$ is the flow stress of the matrix phase in tension and $\dot{\varepsilon}_{0}$ denotes a reference strain-rate. The yield strength $\sigma_{y}$ 
need not be constant (i.e., perfect-plasticity), whereby in the present work it is taken to be a function of the accumulated plastic strain $\varepsilon_{M}^{p}$ in the matrix phase. In the above expression, the exponent $n$ is the inverse of the strain-rate sensitivity parameter and takes values between 1 and $\infty$. The value $n=1$ corresponds to a linearly viscous material, whereby the limit $n \rightarrow \infty$ needs to be considered separately and leads to a rate-independent material response, which is the focus in the present study.

Thus, by considering the limit $n \rightarrow \infty$ in equation (7), we obtain

$$
U\left(\boldsymbol{\sigma} ; s_{\alpha}\right)=\left\{\begin{array}{lc}
0, & \widehat{\sigma}_{e} \leq \sigma_{y}\left(\varepsilon_{M}^{p}\right) \\
\infty, & \text { otherwise }
\end{array}\right.
$$

which directly gives the yield function as

$$
\Phi\left(\boldsymbol{\sigma} ; s_{\alpha}\right)=\widehat{\sigma}_{e}-\sigma_{y}\left(\varepsilon_{M}^{p}\right)
$$

such that the yield condition is defined by $\Phi\left(\boldsymbol{\sigma} ; s_{\alpha}\right)=0$.

\subsubsection{The original Variational formulae (VAR)}

In the original variational method, the effective stress measure $\widehat{\sigma}_{e}$ is given by the explicit expression (Danas, 2008)

$$
\widehat{\sigma}_{e}=\sqrt{\frac{\sigma_{i j} m_{i j k l} \sigma_{k l}}{1-f}}
$$

where the fourth-order tensor $\mathbf{m}$ is written in terms of the microstructural tensor $\mathbf{Q}$, which is defined as

$$
m_{i j k l}^{v a r}=\frac{3}{2} K_{i j k l}+\frac{3 f}{1-f} \lim _{\kappa \rightarrow \infty} \mu Q_{i j k l}^{-1}
$$

Here, the subscript "var" has been used to denote the original result of Ponte Castañeda (1991a), which is a rigorous upper bound of the effective response of the porous material. In the limit $\kappa \rightarrow \infty$ in (11), the tensor $\mathbf{Q}$ becomes a homogeneous function of degree one in $\mu$ and hence the tensor $\mathbf{m}$ is independent of $\mu$. In addition, $\mathbf{Q}$ and consequently $\mathbf{m}$ are functions of the microstructural variables $s_{\alpha}$ and for a non-zero porosity $f$ are both compressible. Note further that when the voids become non-spherical, i.e., when the aspect ratios, defined in (1), take values other than unity, $\mathbf{m}$ becomes anisotropic. The explicit expressions for the evaluation of the microstructural tensor $\mathbf{Q}$ are detailed in the Appendix of Aravas and Ponte Castañeda (2004).

\subsubsection{The modified Variational or modified secant formulae}

The original variational formulation of Ponte Castañeda (1991a), discussed previously, has been found to be sufficiently accurate at low stress triaxialities but tends to overestimate the effective response of the porous material at high stress triaxialities, especially at low porosities. In this connection, following the earlier works of Ponte Castañeda (1991b) and Michel and Suquet (1992), we correct expression (11) by modifying only the hydrostatic part of $\mathbf{m}$, such that

$$
m_{i j k l}^{\text {mvar }}=m_{i j k l}^{v a r}+\left(q_{J}^{2}-1\right) J_{i j p q} m_{p q r s}^{v a r} J_{r s k l}, \quad q_{J}=\frac{1-f}{\sqrt{f} \ln (1 / f)} .
$$

The scalar factor $q_{J}$ brings the yield function (9) into alignment with the spherical shell (or equivalently the "composite sphere assemblage") and the cylindrical shell (or equivalently the "composite cylinder assemblage") solutions when subjected to purely hydrostatic loadings, while preserving standard requirements, such as convexity and smoothness of the yield surface for the entire range of microstructural configurations. Note, however, that the new modified variational model in (12) is not an upper bound but an estimate for the effective behavior of porous materials. Nonetheless, this estimate has the following properties. In one hand, it reproduces the Gurson model in the special case of spherical voids and purely hydrostatic loading, 
while satisfying exactly the variational bound for all range of stress triaxialities and microstructures. In addition, the correction factor $q_{J}$ in (12) brings into alignment the present model with the second-order model of Danas and Ponte Castañeda (2009a) for any choice of the microstructural variables $s_{\alpha}$ in the case of purely hydrostatic loadings.

At this point, it should be mentioned that more complicated interpolation functions could in general be proposed in (12), however, there is risk that the yield surface becomes non-convex for some range of microstructural configurations (e.g., large values of the void aspect ratios). Thus, any modification of the above-prescribed interpolation requires, in general, dependence upon the microstructural variables, such as the porosity and the aspect ratios. However, it will be shown in the following that the interpolation (12) is adequately accurate when compared with the second-order model of Danas and Ponte Castañeda (2009a).

\subsubsection{The spherical and cylindrical shell solutions vs. the VAR and MVAR estimates}

In order to demonstrate that the MVAR recovers the exact spherical and cylindrical shell results, we first consider the special case of spherical voids (i.e., $w_{1}=w_{2}=1$ or $a_{1}=a_{2}=a_{3}$ ) which implies that the constitutive response of the porous material is isotropic. Hence, for purely hydrostatic loadings, the spherical shell yield function becomes (Gurson, 1977; Leblond et al., 1994)

$$
\Phi^{s s h}\left(\sigma_{m} ; f\right)=\frac{3}{2} \frac{1}{\ln (1 / f)}\left|\sigma_{m}\right|-\sigma_{y}
$$

which implies that at yield

$$
\Phi^{s s h}=0 \Rightarrow \frac{\left|\sigma_{m}\right|}{\sigma_{y}}=\frac{2}{3} \ln (1 / f) .
$$

This last exact result is also obtained by using the Gurson (1977) yield function at purely hydrostatic loads, i.e.,

$$
\Phi^{g u r}\left(\sigma_{m} ; f\right)=2 f \cosh \left(\frac{3}{2} \frac{\sigma_{m}}{\sigma_{y}}\right)-1-f^{2} .
$$

Considering the yield condition $\Phi^{g u r}=0$ and solving for $\sigma_{m}$, one obtains (14).

In turn, in the limit of spherical voids and purely hydrostatic loading, the original variational yield function, as obtained by using relations (9), (10) and (11), reads (Danas et al., 2008b)

$$
\Phi_{H}^{v a r}\left(\sigma_{m} ; f\right)=\frac{3}{2} \frac{\sqrt{f}}{1-f}\left|\sigma_{m}\right|-\sigma_{y} .
$$

Solution of (16) at yield gives

$$
\Phi^{v a r}=0 \Rightarrow \frac{\left|\sigma_{m}\right|}{\sigma_{y}}=\frac{2}{3} \frac{1-f}{\sqrt{f}} .
$$

This last equation clearly deviates from the exact shell solution (14), especially for small porosities.

However, the corrected modified variational model proposed in equation (12) gives

$$
\Phi_{H}^{m v a r}\left(\sigma_{m} ; f\right)=\frac{3}{2} q_{J} \frac{\sqrt{f}}{1-f}\left|\sigma_{m}\right|-\sigma_{y}=\frac{3}{2} \frac{1}{\ln (1 / f)}\left|\sigma_{m}\right|-\sigma_{y},
$$

which is identical to equation (13) and hence reproduces the exact result (14).

At this point, it should be noted that under hydrostatic loadings the spherical shell result coincides with the composite sphere assemblage solution of Hashin (1962), and thus it constitutes an exact result for this specific isotropic microstrocture. In addition, the same exact result is recovered by the isotropic infinite-rank laminate microstructure (see Danas et al. (2008b) and Idiart (2008a)). These observations suggest, albeit not in a rigorous manner, that the exact shell result is a very accurate estimate for the effective response of isotropic porous materials.

Similar to the above analysis, one can also consider the limiting case of cylindrical voids with circular cross-section, i.e., the cylindrical shell problem (or equivalently the "composite cylinder assemblage") subjected to in-plane hydrostatic pressure. This case can be readily obtained by allowing any one of the three 
semi-axes $\left(a_{1}, a_{2}\right.$ or $\left.a_{3}\right)$ of the voids tend to infinity, while setting the other two equal. Here, we consider $a_{3} \rightarrow \infty$ and $a_{1}=a_{2}$, while the cylindrical shell is subjected to hydrostatic pressure in the plane $1-2$ with all the out-of-plane components of the deformation tensor set equal to zero (i.e., plane-strain). The resulting yield function for this cylindrical shell becomes then

$$
\Phi^{c s h}\left(\sigma_{\gamma \gamma} ; f\right)=\frac{\sqrt{3}}{2} \frac{1}{\ln (1 / f)}\left|\sigma_{\gamma \gamma}\right|-\sigma_{y}, \quad \gamma=1,2 .
$$

The above result is equivalent to those obtained by the relevant two-dimensional Gurson model (c.f., equation (3.18) in Gurson (1977)) and the two-dimensional infinite-rank laminate microstructures (Danas et al., 2008a; Idiart, 2008b).

On the other hand, considering the limit $a_{3} \rightarrow \infty$ in the original variational method (Danas et al., 2008a), one obtains

$$
\Phi^{v a r}\left(\sigma_{\gamma \gamma} ; f\right)=\frac{\sqrt{3}}{2} \frac{\sqrt{f}}{1-f}\left|\sigma_{\gamma \gamma}\right|-\sigma_{y}, \quad \gamma=1,2
$$

which again deviates from the exact solution (19). However, considering the modified variational model obtained by using the corrected equation (12), one recovers the exact solution (19), i.e.,

$$
\Phi_{H}^{m v a r}\left(\sigma_{\gamma \gamma} ; f\right)=\frac{\sqrt{3}}{2} q_{J} \frac{\sqrt{f}}{1-f}\left|\sigma_{\gamma \gamma}\right|-\sigma_{y}=\frac{\sqrt{3}}{2} \frac{1}{\ln (1 / f)}\left|\sigma_{\gamma \gamma}\right|-\sigma_{y}, \quad \gamma=1,2 .
$$

The fact that the use of the simple scalar correction factor $q_{J}$, defined in relation (12), leads to two exact solutions for two different microstructures is not trivial. It rather suggests that the original variational method comprises the appropriate qualitative features of microstructure evolution, i.e., it predicts the change of the factor 3 (for $a_{1}=a_{2}=a_{3}$ ) in relation (16) to the factor $\sqrt{3}$ (for $\left.a_{3} \rightarrow \infty, a_{1}=a_{2}\right)$ in $(20)$ ).

\subsubsection{The plastic flow rule}

The plastic strain-rate $\boldsymbol{D}^{p}$ is evaluated in terms of the yield function $\Phi$ using the standard normality condition and is given by

$$
D_{i j}^{p}=\dot{\Lambda} N_{i j}, \quad N_{i j}=\frac{\partial \Phi}{\partial \sigma_{i j}}=\frac{m_{i j k l} \sigma_{k l}}{\sqrt{(1-f) \sigma_{p q} m_{p q r s} \sigma_{r s}}}
$$

where $\mathbf{m}=\mathbf{m}^{\text {var }}$ from equation (11) for the VAR model and $\mathbf{m}=\mathbf{m}^{\text {mvar }}$ from equation (12) for the MVAR model. The scalar $\dot{\Lambda} \geq 0$ is the plastic multiplier and is determined from the consistency condition as discussed in Aravas and Ponte Castañeda (2004) (c.f. equation (44) in that manuscript).

\section{Evolution of microstructure}

When porous materials undergo large plastic deformations, the underlying microstructure and therefore the morphological anisotropy of the material evolve. The microstructure evolution, in turn, affects the response of the material itself since the yield condition and the plastic flow rule depend on the current state of the microstructure. Thus, evolution laws for the microstructural state variables $s_{\alpha}$ have to be prescribed. In the current application, we assume that evolution of microstructure occurs only due to plastic deformation of the matrix. In addition, it is important to note that the purpose of homogenization models (such as the MVAR and VAR model discussed in the present study) is the description of the effective behavior in average terms. Therefore, ellipsoidal voids, whose shape and orientation is described by the two aspect ratios $w_{1}$ and $w_{2}$ and the orientation vectors $\mathbf{n}^{(i)}(i=1,3)$, respectively, evolve-on average-to ellipsoidal voids with different shape and orientation. This, in turn, suggests that the average change in shape and orientation of the voids depends only upon the average strain-rate $\boldsymbol{D}^{v}$ and the average spin $\boldsymbol{W}^{v}$ in the vacuous phase. These two tensorial quantities can readily be obtained as a byproduct of the homogenization process as discussed below. Then, the evolution laws for the microstructural variables are obtained simply by appropriate kinematic considerations. 


\subsection{Evolution of the accumulated plastic strain in the matrix and the porosity}

The evolution equation for the accumulated plastic strain in the matrix phase $\varepsilon_{M}^{p}$ is determined by the condition that the macroscopic plastic work $\sigma_{i j} D_{i j}^{p}$ be equal to the corresponding microscopic plastic work $(1-f) \sigma_{y} \dot{\varepsilon}_{M}^{p}$, which implies that (Gurson, 1977)

$$
\dot{\varepsilon}_{M}^{p}=\frac{\sigma_{i j} D_{i j}^{p}}{(1-f) \sigma_{y}}=\dot{\Lambda} \frac{\sigma_{i j} N_{i j}}{(1-f) \sigma_{y}} .
$$

For strain hardening materials, $\sigma_{y}$ is a function of $\varepsilon_{M}^{p}$, which, in general, is to be extracted from experimental uniaxial stress-strain curves. In our work, a rather general strain hardening law for $\sigma_{y}\left(\varepsilon_{M}^{p}\right)$ will be given in the results section.

Any changes of the pores are assumed to be only the result of plastic deformations (Aravas and Ponte Castañeda, 2004) while elastic deformations are considered to have a negligible effect on the evolution of the voids volume fraction. Noting further that the matrix material is plastically incompressible ( $J_{2}$ plasticity), the evolution equation for the porosity $f$ follows easily from the continuity equation and reads

$$
\dot{f}=(1-f) D_{k k}^{p}=\dot{\Lambda}(1-f) N_{k k} .
$$

We point out that void nucleation is not considered in the above relation but can be readily included by proper modification of (24)(e.g., Needleman and Rice (1978); Chu and Needleman (1980); Tvergaard (1990)).

\subsection{Evolution of the aspect ratios}

The evolution of the aspect ratios $w_{1}$ and $w_{2}$, describing the shape of the voids, is given in terms of the average strain-rate in the vacuous phase $\boldsymbol{D}^{v}$, which is given in terms of the fourth-order concentration (or localization) tensor $\mathbf{A}$, such that

$$
D_{i j}^{v}=A_{i j k l} D_{k l}^{p}=\dot{\Lambda} A_{i j k l} N_{k l}, \quad A_{i j k l}=\frac{1}{f}\left(I_{i j k l}-\frac{1}{2} K_{i j p q} m_{p q k l}^{-1}\right) .
$$

Here, $\mathbf{m}=\mathbf{m}^{v a r}$ is given from equation (11) for the VAR model and $\mathbf{m}=\mathbf{m}^{\text {mvar }}$ from equation (12) for the MVAR model. In the above relation, we have made the assumption that $\boldsymbol{D}^{v}$ depends only on the plastic strain-rate $\boldsymbol{D}^{p}$ which is consistent with the assumption made in the beginning of the section stating that elastic deformations are very small and hence do not contribute to the evolution of the microstructural variables.

The evolution law for the aspect ratios is obtained by using standard kinematics and the definition $w_{s}=a_{3} / a_{s}(\mathrm{~s}=1,2)$, such that

$$
\dot{w}_{s}=\alpha_{w} w_{s}\left(\frac{\dot{a_{3}}}{a_{3}}-\frac{\dot{a_{s}}}{a_{s}}\right)=\alpha_{w} w_{s}\left(n_{i}^{(3)} n_{j}^{(3)}-n_{i}^{(s)} n_{j}^{(s)}\right) D_{i j}^{v}=\dot{\Lambda} \alpha_{w} w_{s}\left(n_{i}^{(3)} n_{j}^{(3)}-n_{i}^{(s)} n_{j}^{(s)}\right) A_{i j k l} D_{k l}^{p},
$$

(no sum on $s=1,2$ ). The scalar factor $\alpha_{w}$ has been introduced in the last expression in a heuristic manner in order to enhance the accuracy of the evolution of the aspect ratios. The reason we introduce this factor is related to the findings of Danas (2008) and Danas and Ponte Castañeda (2009b), who showed that the original variational method and consequently the modified variational model of the present work tend to underestimate the evolution of the void shape at low stress triaxialities. In general, $\alpha_{w}$ is a free parameter that can be calibrated from experiments. In the present study (i.e., rate-independent plasticity with $n \rightarrow \infty)$, we choose the value $\alpha_{w}=7 / 4$. This is done in order to bring the modified variational model in good agreement (c.f. section 5.3) with the recent second-order model (Danas and Ponte Castañeda, 2009a), which was shown to be very accurate when compared with unit-cell finite element calculations in all range of stress triaxialities considered (see Danas (2008) and Danas and Ponte Castañeda (2009b)). It should be noted, however, that in the case of rate-dependent porous materials, $a_{w}$ is expected to be a function of the strain-rate sensitivity $n$ since for $n=1$ (linearly viscous material), the value $a_{w}=1$ leads to the exact solution in the dilute limit $(f \rightarrow 0)$, and hence no modifications are needed in that case. In addition, one 
could also let $a_{w}$ be a heuristic function of the stress triaxiality in order to capture the counterintuitive elongation of the void shape transversely to the maximum principal stress at very high stress triaxialities and dilute porosities (see relevant discussion in Budiansky et al. (1982), Fleck and Hutchinson (1986) and Danas and Ponte Castañeda (2009b)) but such an attempt is not pursued here.

\subsection{Evolution of the orientation vectors}

The evolution of the orientation vectors $\mathbf{n}^{(i)}(i=1,3)$ depends upon the average strain-rate $\boldsymbol{D}^{v}$ (see (25)) and the average spin $\boldsymbol{W}^{v}$ in the vacuous phase, which, in turn, are given in terms of the overall plastic strain-rate $\boldsymbol{D}^{p}$ and the overall spin $\boldsymbol{W}$. Thus, first, it is helpful to write the average spin in the vacuous phase as (Kailasam and Ponte Castañeda, 1998)

$$
W_{i j}^{v}=W_{i j}-C_{i j k l} D_{k l}^{v}=W_{i j}-C_{i j k l} A_{k l m n} D_{m n}^{p}, \quad C_{i j k l}=-(1-f) \Pi_{i j p q} A_{p q k l},
$$

where $\boldsymbol{\Pi}$ is the fourth-order Eshelby (1957) rotation tensor and depends on the aspect ratios $w_{1}$ and $w_{2}$ as well as on the orientation vectors $\mathbf{n}^{(i)}(i=1,3)$. The tensor $\boldsymbol{\Pi}$ is antisymmetric with respect to the two first indices and symmetric with respect to the last two, i.e., $\Pi_{i j k l}=-\Pi_{j i k l}=\Pi_{i j l k}$. The evaluation of the $\Pi$ tensor is detailed in the Appendix of Aravas and Ponte Castañeda (2004).

The evolution of the orientation vectors $\mathbf{n}^{(i)}$ is determined by the spin of the Eulerian axes of the ellipsoidal voids, or "microstructural" spin $\boldsymbol{\omega}$, via

$$
\dot{n}_{i}^{(k)}=\omega_{i j} n_{j}^{(k)}, \quad k=1,2,3 .
$$

The microstructural spin $\boldsymbol{\omega}$ is related to the average spin in the void, $\boldsymbol{W}^{v}$, and the average strain-rate in the void, $\boldsymbol{D}^{v}$, by the well-known kinematical relation, which is written in direct notation as (Hill, 1978; Aravas and Ponte Castañeda, 2004)

$$
\omega_{i j}=W_{i j}^{v}+\frac{1}{2} \sum_{\substack{r, s=1 \\ r \neq s \\ w_{r} \neq w_{s}}}^{3} \frac{w_{r}^{2}+w_{s}^{2}}{w_{r}^{2}-w_{s}^{2}}\left[\left(n_{p}^{(r)} n_{q}^{(s)}+n_{q}^{(s)} n_{p}^{(r)}\right) D_{p q}^{v}\right] n_{i}^{(r)} n_{j}^{(s)}, \quad w_{3}=1 .
$$

The special case in which at least two aspect ratios are equal is discussed in detail later in this section.

The evaluation of the Jaumann rate (which is an objective rate) of the orientation vectors $\mathbf{n}^{(i)}$, denoted by $\stackrel{\nabla}{\mathbf{n}}^{(k)}(k=1,2,3)$, is related to the standard time derivative of relation $(28)$ by

$$
\stackrel{\nabla}{n}_{i}^{(k)}=\dot{n}_{i}^{(k)}-W_{i j} n_{j}^{(k)}=\left(\omega_{i j}-W_{i j}\right) n_{j}^{(k)}, \quad k=1,2,3 .
$$

The last equation can be written in terms of the plastic spin (Dafalias, 1985), which is defined as the spin of the continuum relative to the microstructure, as follows

$$
\stackrel{\nabla}{n}_{i}(k)=-W_{i j}^{p} n_{j}^{(k)}, \quad W_{i j}^{p}=W_{i j}-\omega_{i j}
$$

Combining the above equations, the plastic spin $\boldsymbol{W}^{p}$ can be written as

$$
W_{i j}^{p}=\dot{\Lambda} \Omega_{i j}^{p}, \quad \Omega_{i j}^{p}=C_{i j k l} N_{k l}-\frac{1}{2} \sum_{\substack{r, s=1 \\ r \neq s \\ w_{r} \neq w_{s}}}^{3} \frac{w_{r}^{2}+w_{s}^{2}}{w_{r}^{2}-w_{s}^{2}}\left[\left(n_{p}^{(r)} n_{q}^{(s)}+n_{q}^{(s)} n_{p}^{(r)}\right) D_{p q}^{v}\right] n_{i}^{(r)} n_{j}^{(s)},
$$

where $\boldsymbol{D}^{v}$ is given by (25).

At this point, we point out that special care needs to be taken for the computation of the spin of the Eulerian axes in the case of a spherical void, i.e., when $w_{1}=w_{2}=w_{3}=1$, as well as for a spheroidal void, i.e., when $w_{1}=w_{2} \neq w_{3}=1$ or $w_{1} \neq w_{2}=w_{3}=1$ or $w_{1}=w_{3}=1 \neq w_{2}$. More specifically, when two of the aspect ratios are equal, for instance $w_{1}=w_{2}$, the material becomes transversely isotropic about the 
$\mathbf{n}^{(3)}$-direction, and thus the component $\Omega_{12}^{p}$ becomes indeterminate. Since the spin $\Omega_{12}^{p}$ is inconsequential in this case, it can be set equal to zero (Aravas, 1992), which implies that $\omega_{12}=\Omega_{12}$. This notion can be applied whenever the shape of the void is spheroidal, in any given orientation. Following a similar line of thought, when the voids are spherical $\left(w_{1}=w_{2}=w_{3}=1\right)$ the material is isotropic so that $\mathbf{\Omega}^{p}=\mathbf{0}, \stackrel{\nabla}{\mathbf{n}}^{(k)}=0$ and $\dot{\mathbf{n}}^{(k)}=\boldsymbol{W} \mathbf{n}^{(k)}(k=1,2,3)$.

\section{Results for the instantaneous effective response and evolution of microstructure}

This section deals with the application of the modified variational model (MVAR) to predict the instantaneous effective response and the evolution of microstructure under finite deformations of a porous material subjected to purely triaxial loading conditions so that the orientation vectors of the voids remain fixed and aligned with the laboratory frame axes. We first define the stress triaxiality and Lode parameters as alternative stress measures of the principal stress components. In the following, we construct yield surfaces for various microstructural configurations, both isotropic and anisotropic, in order to investigate the effect of void shape upon the instantaneous effective response of the porous material. This section ends with the study of the effect of the Lode parameter and the stress triaxiality on the microstructure evolution at finite deformations. For comparison purposes, the MVAR model is compared with the original variational bound (VAR) (Ponte Castañeda, 1991a), the second-order model (SOM) (Danas and Ponte Castañeda, 2009a) and the Gurson (1977) model (GUR). It is recalled that while the MVAR, VAR and SOM models belong to the same family of homogenization models and hence include void shape effects, the GUR model is based on the spherical shell analysis and hence remains isotropic (i.e., voids remain spherical) during the entire deformation process.

\subsection{Triaxial loading conditions: Stress triaxiality and Lode parameter}

This subsection discusses the loading conditions and the associated stress measures used to distinguish between hydrostatic loading and different shear stress states. In the context of purely triaxial loading conditions, one can define alternative stress measures other than the principal stresses that are more appropriate for dilatational plasticity of porous materials. The three alternative measures are the hydrostatic (or mean) stress, $\sigma_{m}$, the von Mises equivalent (or effective) stress, $\sigma_{e}$, and the third invariant of the stress deviator, $J_{3}$, defined as

$$
\sigma_{m}=\frac{\sigma_{k k}}{3}, \quad \sigma_{e}=\sqrt{3 J_{2}}=\sqrt{\frac{3}{2} s_{i j} s_{i j}}, \quad J_{3}=\operatorname{det}\left(s_{i j}\right),
$$

where $s_{i j}=\sigma_{i j}-\sigma_{m} \delta_{i j}$ is the stress deviator. Using these definitions, we can readily define the stress triaxiality, $X_{\Sigma}$, and Lode parameter, $L$, or equivalently the Lode angle, $\theta$, via the following expressions

$$
X_{\Sigma}=\frac{\sigma_{m}}{\sigma_{e}}, \quad L=-\cos 3 \theta=-\frac{27}{2} \frac{J_{3}}{\sigma_{e}^{3}} .
$$

By definition, the range of values for the $X_{\Sigma}$ and $L$, (or $\left.\theta\right)^{1}$ are

$$
-\infty<X_{\Sigma}<\infty, \quad \text { and } \quad-1 \leq L \leq 1 \quad \text { or } \quad 0 \leq \theta \leq \pi / 3
$$

In order to illustrate the connection of $X_{\Sigma}$ and $L$ with the standard stress components, we consider the principal stresses $\sigma_{1}=\sigma_{11}, \sigma_{2}=\sigma_{22}$ and $\sigma_{3}=\sigma_{33}\left(\sigma_{i j}=0\right.$ for $\left.i \neq j\right)$ that are aligned with the laboratory frame axes, $\mathbf{e}^{(1)}, \mathbf{e}^{(2)}$ and $\mathbf{e}^{(3)}$, respectively. Then, relations (34) can be used to express the principal stresses as functions of $X_{\Sigma}, \sigma_{e}$ and $\theta$, such that

$$
\frac{3}{2 \sigma_{e}}\left\{\sigma_{1}, \sigma_{2}, \sigma_{3}\right\}=\left\{-\cos \left(\theta+\frac{\pi}{3}\right),-\cos \left(\theta-\frac{\pi}{3}\right), \cos \theta\right\}+\frac{3}{2} X_{\Sigma}\{1,1,1\}
$$

\footnotetext{
${ }^{1}$ In the literature, other definitions of the Lode parameter have also been used. In particular, using the very common definition for the Lode parameter $\mu=\left(2 \sigma_{1}-\sigma_{3}-\sigma_{2}\right) /\left(\sigma_{3}-\sigma_{2}\right)$, one finds readily that $\sqrt{3} L=\mu$ in expression $(34)_{2}$.
} 
a)

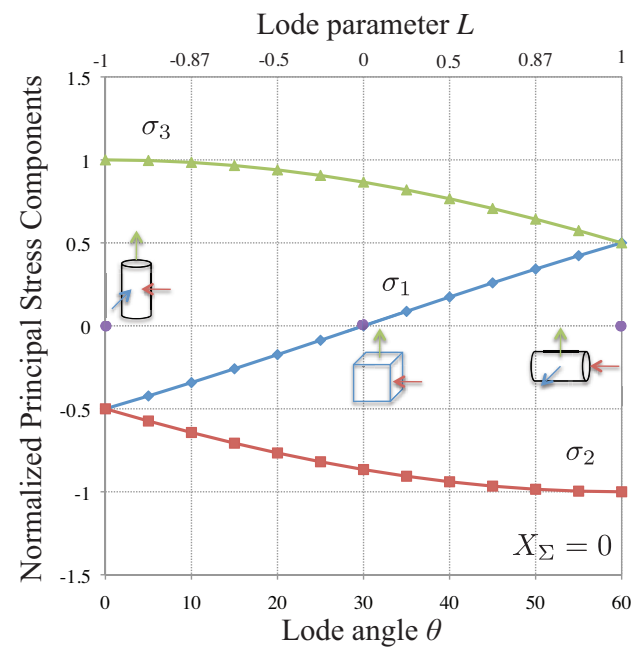

b)

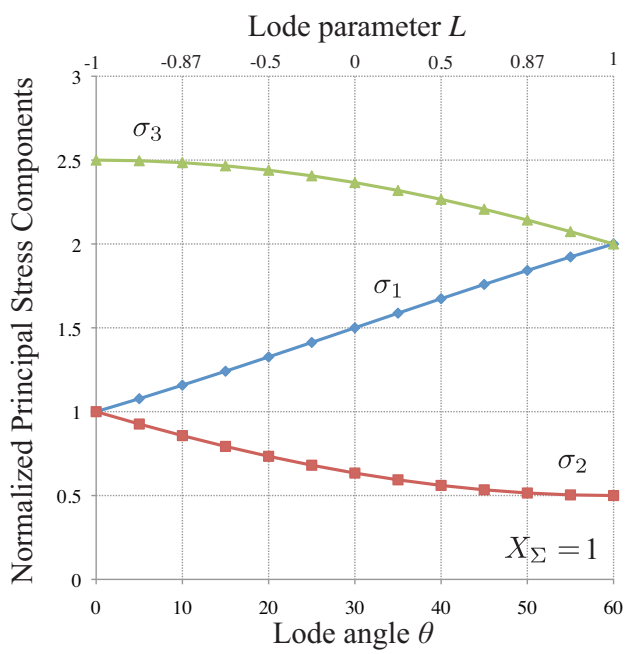

Figure 2: Normalized principal stresses $3\left\{\sigma_{1}, \sigma_{2}, \sigma_{3}\right\} / 2 \sigma_{e}$, as a function of the Lode angle $\theta$ or equivalently the Lode parameter $L$. Parts (a) and (b) correspond to stress triaxialities $X_{\Sigma}=0$ and $X_{\Sigma}=1$, respectively.

It should be noted, here, that if initially anisotropic materials are considered (e.g., arbitrary ellipsoidal void shapes) then one should consider $0 \leq \theta<2 \pi$ in order to attain all possible values for the principal stress components (see for instance $\Pi$-plane graphs in Danas and Ponte Castañeda (2009b)).

Figure 2 shows the normalized principal stresses defined in (36) as a function of the Lode parameter $L$ and Lode angle $\theta$ for (a) $X_{\Sigma}=0$ and (b) $X_{\Sigma}=1$. It is obvious from Fig. 2a that for $L=-1$ or $\theta=0$, the stress state is axisymmetric with one positive and two negative stresses (axisymmetric tension). On the other end, when $L=1$ or $\theta=\pi / 3$, the stress state is also axisymmetric but with two positive and one negative stresses (biaxial tension with axisymmetric compression). Note that these two different axisymmetric states lead to different evolution of the underlying microstructure and therefore to different overall responses as the deformation progresses. When, $L=0$ or $\theta=\pi / 6$, the stress state is in-plane shear with one stress identically equal to zero (e.g., plane stress state). The rest of the states are between axisymmetric and in-plane shear states. It should be noted that when the stress triaxiality is nonzero then the principal stresses are simply translated by a constant either upwards for $X_{\Sigma}>0$, as shown in Fig. $2 \mathrm{~b}$ for $X_{\Sigma}=1$, or downwards for $X_{\Sigma}<0$ (not shown here for brevity). Note also that $\left|X_{\Sigma}\right| \rightarrow \infty$ and $X_{\Sigma}=0$ correspond to purely hydrostatic and purely deviatoric loadings, respectively.

\subsection{Yield surfaces}

In this section, we present results for the instantaneous effective response of porous materials comprising voids with spherical and non-spherical shapes. First, we examine the accuracy of the estimates obtained by the present MVAR model and its ability to recover the Gurson result in the purely hydrostatic limit. Second, we examine the effect of the void shape on the resulting yield surface of the porous material.

\subsubsection{Isotropic microstructures}

Figure 3 shows yield surfaces for spherical voids (i.e., $w_{1}=w_{2}=1$ ) as predicted by a) the modified variational model (MVAR), b) the variational bound (VAR), c) the second-order model (SOM) of Danas et al. (2008b) and d) the Gurson (1977) model (GUR). The porosity is set equal to $f=1 \%$ and the loading is axisymmetric (i.e., $L=-1$ or $\theta=0$ ). The MVAR model is identical to the VAR bound at zero stress triaxiality (i.e., purely deviatoric load $\sigma_{m}=0$ ) and deviates from the bound with increasing $\sigma_{m}$. In the purely hydrostatic limit (i.e., $\sigma_{e} \rightarrow 0$ ), the MVAR model attains the analytical spherical shell solution and thus coincides with the SOM and Gurson models. For intermediate values of the mean stress 
(i.e., $1<\sigma_{m}<2.8$ ), however, the difference between the MVAR and GUR models is significant. In this case, the MVAR agrees well with the SOM model, which has been shown to give very accurate estimates when compared with numerical high-rank laminate microstructures (see Danas et al. (2008b) for a detailed comparison). At this point, it is worth noting that for the axisymmetric loading considered here the SOM model is not symmetric with respect to the $\sigma_{m}=0$ vertical axis implying that for purely deviatoric stress loads the corresponding strain tensor (extracted by using the normal to the SOM yield surface) exhibits a small hydrostatic part, as already discussed in Danas et al. (2008b). This is attributed to the fact that the isotropic SOM yield surface depends also on the third invariant of the stress tensor, i.e., $J_{3}$. This is not the case for the rest of the models, which have a quadratic character and hence depend only on the first two invariants, the mean stress $\sigma_{m}$ and the von Mises stress $\sigma_{e}$. Nonetheless, the dependence of the more accurate SOM isotropic yield surface upon the third invariant of the stress is rather negligible, especially for small porosities and, as will be discussed later in this section, it is of minor importance when compared to the corresponding void shape effects at a given level of porosity.

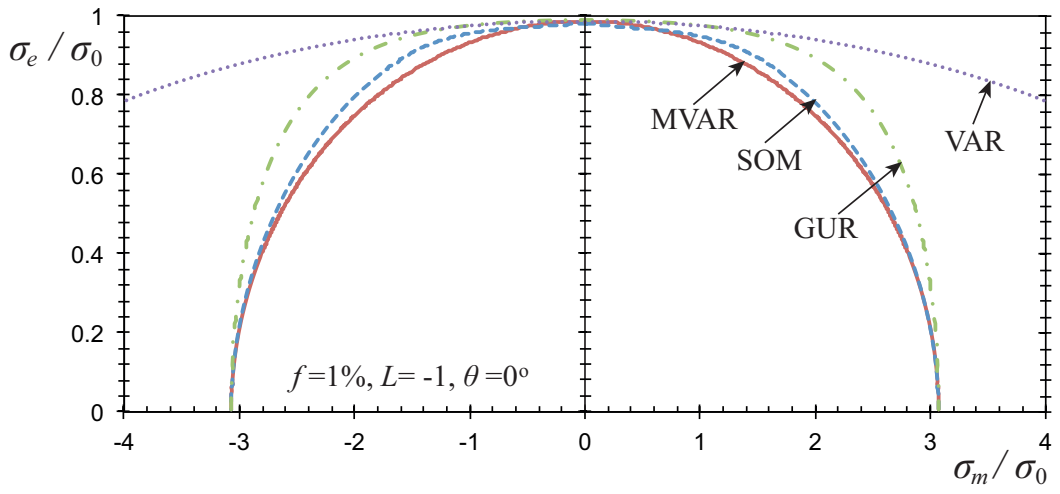

Figure 3: Yield surfaces in the $\sigma_{e}-\sigma_{m}$ plane for isotropic microstructures (i.e., $w_{1}=w_{2}=1$ ). Comparison between the various models (modified variational MVAR, variational VAR, second-order SOM, Gurson GUR) for $f=1 \%$ and $L=-1($ or $\theta=0)$.

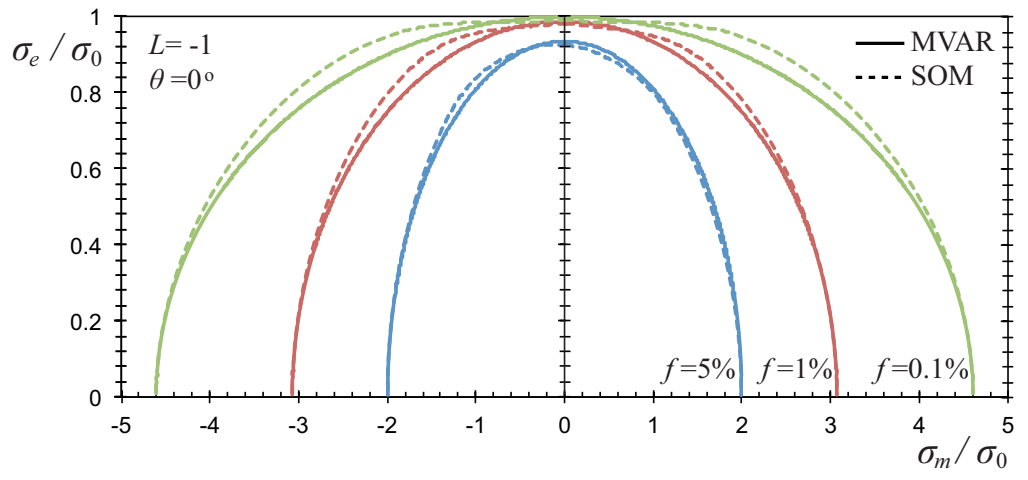

Figure 4: Yield surfaces in the $\sigma_{e}-\sigma_{m}$ plane for isotropic microstructures (i.e., $w_{1}=w_{2}=1$ ). Comparison between the modified variational MVAR and the second-order SOM models for three different porosities $f=0.1,1,5 \%$ and $L=-1($ or $\theta=0)$.

In Fig. 4, we compare the MVAR and SOM isotropic yield surfaces for three different porosities, $f=$ $0.1,1,5 \%$. The main observation is that the simple modification of the original variational bound, introduced 
in equation (12), leads to a satisfactory agreement between the MVAR and the SOM predictions for a large range of porosities. The largest difference between the MVAR and the SOM estimates is found for smaller porosities $(f=0.01 \%)$ and moderate values of the mean stress $\left(2<\sigma_{m}<4\right)$. In addition, the asymmetry of the SOM yield surface with respect to the $\sigma_{m}=0$ vertical axis diminishes with decreasing porosity.

At this point, it should be noted that while the differences between the MVAR and the SOM yield surfaces are relatively small, the differences in the normals $\partial \Phi / \partial \boldsymbol{\sigma}$, (which are essential for the evolution of the microstructure) are not necessarily close (in relative terms). In fact, the MVAR model tends to give more compliant estimates than the SOM model especially for stress triaxialities in the order of $1 \sim 2$.

\subsubsection{Anisotropic microstructures}

Figure 5 shows MVAR and SOM yield surfaces for a) spherical voids $\left(w_{1}=w_{2}=1\right)$, b) prolate voids $\left.\left(w_{1}=w_{2}=5\right), \mathrm{c}\right)$ oblate voids $\left(w_{1}=w_{2}=0.2\right)$ and $\left.\mathrm{d}\right)$ arbitrary ellipsoidal voids $\left(w_{1}=5, w_{2}=0.2\right)$. The porosity is set equal to $f=1 \%$, whereas the loading is axisymmetric along the $x_{3}$-axis $(L=-1)$. This implies that in the present case of prolate and oblate voids, which are also axisymmetric with respect to the $x_{3}$-axis, the corresponding material response is transversely isotropic. In contrast, for the more general case with the ellipsoidal void shape $\left(w_{1}=5, w_{2}=0.2\right)$ the response of the porous material is orthotropic with the axes of orthotropy coinciding with the ellipsoidal void axes. The main observation in Fig 5 is that non-spherical void shapes have a dramatic influence on the yield surface of the porous material as predicted by both the MVAR and the SOM models. First, the slopes of the yield surfaces depend strongly on the void shape. For instance, a porous material with ellipsoidal voids $\left(w_{1}=5, w_{2}=0.2\right)$ is stiffer than that with oblate voids $\left(w_{1}=w_{2}=0.2\right)$ in the regime of $-0.6<\sigma_{m}<1.2$, whereas it becomes more compliant for the rest of the mean stress values. In addition, for the same value of porosity, non-spherical void shapes lead to a significantly more compliant response at high values of the mean stress, especially in the case of oblate and arbitrary ellipsoidal voids. Moreover, it is evident from this figure that arbitrary ellipsoidal shapes $\left(w_{1}=5, w_{2}=0.2\right)$ lead to very different responses when compared with spheroidal shapes $\left(w_{1}=w_{2}=0.2\right.$ or $\left.w_{1}=w_{2}=0.2\right)$.

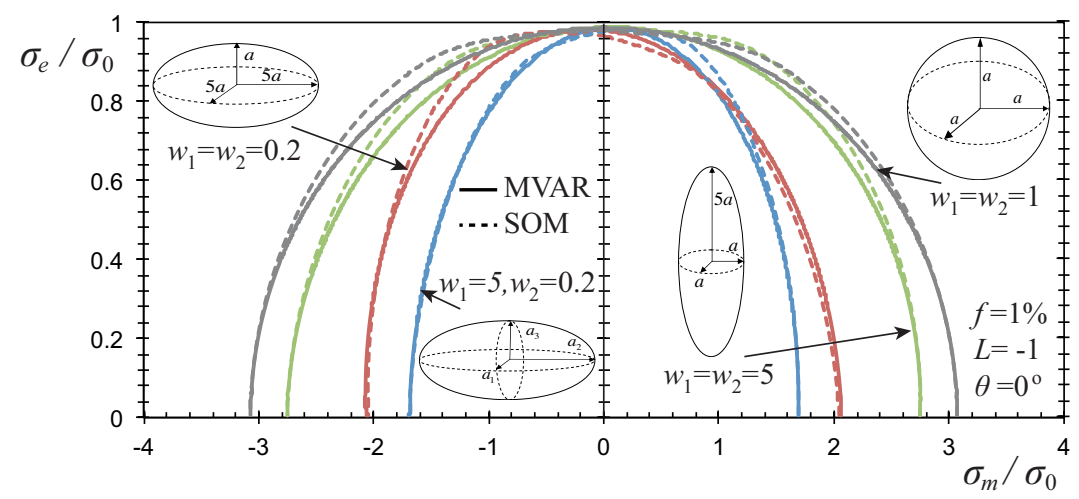

Figure 5: Yield surfaces in the $\sigma_{e}-\sigma_{m}$ plane for isotropic (spherical voids $w_{1}=w_{2}=1$ ) and anisotropic microstructures; prolate voids $w_{1}=w_{2}=5$, oblate voids $w_{1}=w_{2}=0.2$ and ellipsoidal voids $w_{1}=5, w_{2}=0.2$. Comparison between the modified variational MVAR and the second-order SOM models for $f=1 \%$ and $L=-1$ (or $\theta=0$ ).

It should be mentioned at this point that a series of additional triaxial loading conditions have also been considered and the MVAR has been found to be in good agreement (similar to the one observed in the previous results) with the corresponding SOM estimates. However, no such results are shown here for brevity. 


\subsection{Stress-strain response and evolution of microstructure under finite deformations}

In this section, the objective is to investigate the effects of the stress triaxiality $X_{\Sigma}$ and Lode parameter $L$ (or Lode angle $\theta$ ) on the macroscopic response and microstructure evolution of porous elasto-plastic materials subjected to triaxial loading conditions and compare the relevant estimates given by the modified variational model (MVAR), the second-order model (SOM) (Danas and Ponte Castañeda, 2009a) and the Gurson (1977) model (GUR). Given the fact that a maximum stress is expected, the strain rate $D_{33}$ is prescribed, together with the values of $X_{\Sigma}$ and $L$, which will serve to determine all three (principal) stresses, $\sigma_{1}, \sigma_{2}$ and $\sigma_{3}$, as well as the evolution of the microstructural variables, the porosity $f$, and the average aspect ratios, $w_{1}$ and $w_{2}$, as functions of time $t$. No rotation of the voids is considered in this stage, so that the principal axes of the voids coincide with the laboratory frame during the entire deformation process, i.e., $\mathbf{n}^{(i)}=\mathbf{e}^{(i)}(i=1,2,3)$. For convenience, the total equivalent strain $\varepsilon_{e}=\int \sqrt{\frac{2}{3} D_{i j}^{\prime} D_{i j}^{\prime}} \mathrm{d} t$, with $D_{i j}^{\prime}$ denoting the strain-rate deviator, is used as a time-like variable, whereas the overall von Mises equivalent stress $\sigma_{e}$ is considered as the main stress measure for the characterization of the macroscopic response in the following results instead of the individual stress components. Because of the special loading conditions imposed, the maximum on the $\sigma_{e}$ versus $\varepsilon_{e}$ plots will correspond exactly to a vanishing hardening rate of the porous material, indicating a possible instability under stress-controlled loading conditions. Furthermore, it should be emphasized, that as a consequence of the very small magnitude of the overall elastic strains, the difference between the overall total strain and the overall plastic strain is very small for all practical purposes.

\subsubsection{Material parameters and initial conditions}

The Young's modulus and Poisson's ratio of the matrix phase are taken to be $E=200 \mathrm{GPa}$ and $\nu=0.3$, respectively, and the matrix phase to exhibit isotropic strain hardening following the law ${ }^{2}$

$$
\sigma_{y}\left(\varepsilon_{M}^{p}\right)=\sigma_{0}\left(1+\frac{\varepsilon_{M}^{p}}{\varepsilon_{0}}\right)^{N}, \quad \varepsilon_{0}=\frac{\sigma_{0}}{E} .
$$

In this expression, $\sigma_{0}$ and $\varepsilon_{0}$ denote the initial yield stress and yield strain of the matrix material (i.e., the material with $f=0$ ), and $N \leq 1$ is the strain hardening exponent. Typical values for these parameters are $\sigma_{0}=200 \mathrm{MPa}$ and $N=0.1$, which will be used throughout this section.

The matrix phase is taken to be initially unloaded with zero accumulated plastic strain $\varepsilon_{M}^{p}=0$, while the voids are initially spherical with $w_{1}=w_{2}=1$. The initial porosity is taken to be $f_{0}=1 \%$. It should be noted that the macroscopic response of the porous material at large deformations is strongly affected by the hardening exponent $N$, the initial porosity $f$ and the initial aspect ratios $w_{1}$ and $w_{2}$, but carrying out a parametric study with respect to those parameters is beyond the scope of this work and the reader is referred to the recent work of Danas and Ponte Castañeda (under review) for more details. On the contrary, the dependence of response upon the Young's modulus, and Poisson's ratio has been found to be weak at large deformations.

In the following, we show results for two representative values of stress triaxialities, $X_{\Sigma}=0.1,1$, and four of the Lode parameter, $L=-1,-0.5,0,1$ (or Lode angle $\theta=0,20^{\circ}, 30^{\circ}, 60^{\circ}$ respectively).

\subsubsection{Low stress triaxiality}

Figure 6 shows MVAR, SOM and GUR plots of (a) the equivalent stress $\sigma_{e}$, (b) the porosity $f$, and the aspect ratios (c) $w_{1}$ and (d) $w_{2}$, as a function of the equivalent strain $\varepsilon_{e}$, for the aforementioned values of the Lode parameter and a low value of the stress triaxiality $\left(X_{\Sigma}=0.1\right)$. The main observation in Fig. 6a is that the Lode parameter strongly affects the onset of softening (i.e., maximum load) of the porous material. In the case of axisymmetric tensile loadings $(L=-1)$, the stress increases following the prescribed strain hardening law of the matrix phase $(N=0.1$ here) for all models. On the other hand, for $L=-0.5,0$,

\footnotetext{
${ }^{2}$ It should be noted here that any hardening law for the matrix phase involving temperature effects or different non-monotonic strain hardening stages can be readily taken into account. However, the simple isotropic model will suffice for the purposes of this work.
} 
and 1 , we observe sudden drops in $\sigma_{e}$ at different levels of the total strain $\varepsilon_{e}$, indicating a sudden loss in the load-carrying capacity of the material, as predicted by both the MVAR and the SOM which are found to be in good agreement. In contrast, the GUR model (almost coincides with the MVAR and SOM for $L=-1$ ) does not exhibit any dependence on the Lode parameter and hence predicts no softening at such low triaxiality.

a)

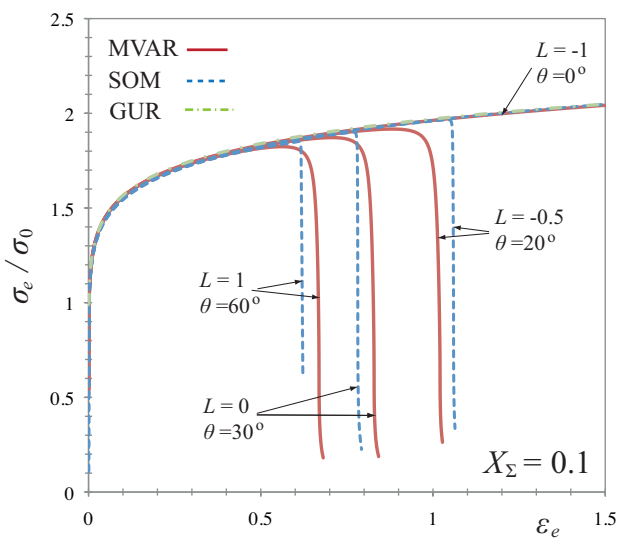

c)

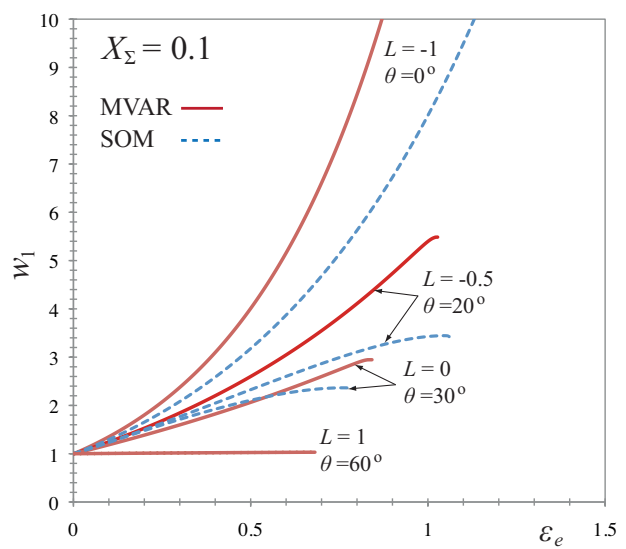

b)

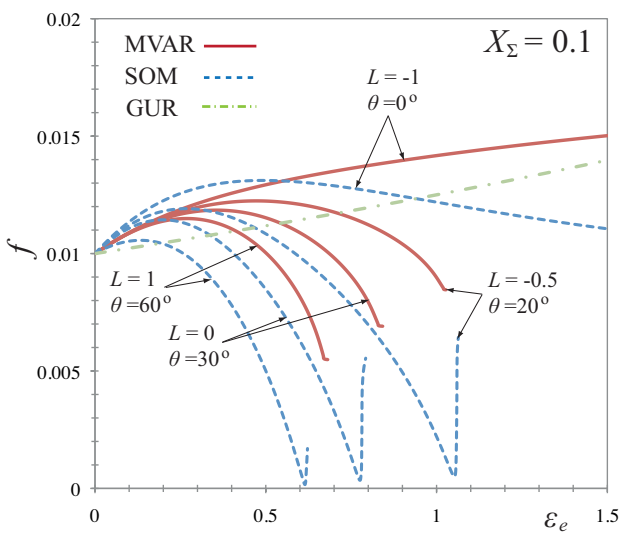

d)

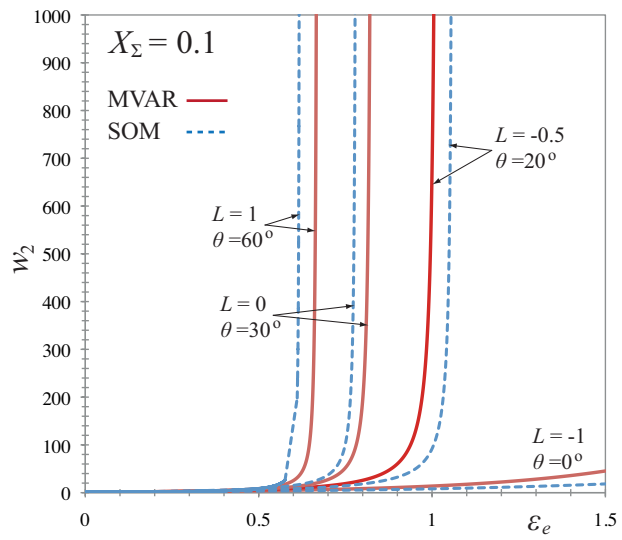

Figure 6: Plots of the MVAR, SOM and GUR estimates for (a) the equivalent stress $\sigma_{e}$, (b) the porosity $f$, and the aspect ratios (c) $w_{1}$ and (d) $w_{2}$ as a function of the equivalent strain $\varepsilon_{e}$, for a low value of the stress triaxiality $\left(X_{\Sigma}=0.1\right)$ and four values of the Lode parameter. The influence of the Lode parameter is dramatic at low triaxialities mainly due to the extremely sharp evolution of the aspect ratio $w_{2}$ in (d). The strain hardening exponent is $N=0.1$ and the initial porosity $f_{0}=1 \%$.

The mechanism leading to this sharp stress drop is directly related to the microstructure evolution. Thus, plots for the evolution of $f, w_{1}$ and $w_{2}$ are provided in Figs. 6 (b), (c) and (d), respectively, as a function of the equivalent strain $\varepsilon_{e}$. In part (b), we observe an overall reduction in the porosity $f$ as a function of $\varepsilon_{e}$ for both the MVAR and SOM models, except in the case of the MVAR prediction for $L=-1$. In contrast, the GUR model predicts a monotonic increase of $f$ with increasing straining. Nonetheless, this porosity increase is not sufficient to overcome the matrix hardening in order to lead to overall softening of the material, as has been observed in Fig. 6a. Therefore, the corresponding stress drop observed in part (a) for both the MVAR 
and SOM estimates cannot be associated with porosity increase, and the only microstructural variables that can possibly affect the overall response of the porous material are the aspect ratios, $w_{1}$ and $w_{2}$.

As shown in part (c), $w_{1}$ can become rather large for $L=-1$, for both the MVAR and the SOM models (GUR model includes no void shape effects), but remain below the value of 5 for $L>-0.5$. On the other hand, as shown in part (d), $w_{2}$ increases very fast for all values of $L$ used here except for $L=-1$. In particular, for $L=1$ (corresponding to axisymmetric compression along the $x_{2}$ direction, see Fig. 2a), $w_{1}=1$, while $w_{2}$ blows up at a certain "critical" value of $\varepsilon_{e}$ for both the MVAR and the SOM models. This implies that a void collapse mechanism (i.e., flattened cracks lying in the $x_{1}-x_{3}$ plane) is developed in the $x_{2}$ direction with increasing strain, while the material becomes locally orthotropic with the axes of orthotropy coinciding with the void axes.

To clarify this failure mechanism further, it is recalled here that the aspect ratios serve to denote both the shape of the voids as well as the shape of their distribution function (see also Danas and Ponte Castañeda (under review)). Hence, as $a_{2} \rightarrow 0$ and $a_{1}=a_{3} \rightarrow \infty$ both the shape of the voids and the shape of their distribution function become extremely flat in the $x_{1}-x_{3}$ plane. This observation together with the fact that the porosity is small but finite, implies that the pores grow without a bound in the $x_{1}-x_{3}$ plane, eventually linking up to form "layers" of pores in the solid material, which can be associated with void coalescence in that plane and subsequent loss of the load-carrying capacity of the material in the transverse direction. This, of course, implies that the corresponding yield surface of the porous material shrinks to zero. In addition, after the maximum load occurs, the porosity tends to grow again, especially in the SOM model, which is a direct consequence of the applied stress (fixed triaxiality and Lode angle) loading conditions and the strong orthotropy developed due to the extreme void elongations in this case. Such a failure mechanism is consistent with the "flat" dimples observed in the experimental results of Barsoum and Faleskog (2007a) at low stress triaxialities. For other values of $L$ with $-1<L<1$, essentially the same mechanism is observed except that in this case the pores also change shape in the collapse plane $\left(x_{1}-x_{3}\right)$. However, as can be seen in Figs. 6a and d, the effect becomes more pronounced as the value of $L$ increases from -1 toward +1 . At the extreme value of $L=-1$, the shape of the pores is constrained to remain prolate, and this kinematic restriction prevents collapse of the pores, explaining the lack of a maximum stress in this case.

\subsubsection{High stress triaxiality}

Figure 7 shows MVAR, SOM and GUR plots of $\sigma_{e}, f, w_{1}$ and $w_{2}$ as a function of the equivalent strain $\varepsilon_{e}$, for the aforementioned values of the Lode parameter $L$ and for a high stress triaxiality $\left(X_{\Sigma}=1\right)$. The main result is that the effect of the Lode parameter on the overall mechanical response of the porous material is negligible, as can be seen in Fig. 7a, since all the $\sigma_{e}-\varepsilon_{e}$ curves almost coincide for the largest range of the applied strain. All models exhibit a maximum stress at rather low strains and then smooth but significant softening as the deformation progresses. Specifically, the MVAR shows the most compliant response among the models shown here, while the GUR prediction is the stiffest one. This is consistent with the observations made in the discussion of the yield surfaces in section 5.2, where it was found that the MVAR is, in most of the cases, the more compliant of all the models considered.

As before, the fact that the stress-strain curve is independent of the Lode parameter at $X_{\Sigma}=1$ is easily explained by referring to Fig. 7b, where the increase of porosity is significant for all values of the Lode parameter $(L=-1,-0.5,0,1)$. The MVAR model predicts the highest increase in $f$, which is consistent with the fastest drop in $\sigma_{e}$ in Fig. 7a, and vice-versa for the corresponding GUR estimate. In addition, by observation of parts (c) and (d), we note that the void shape still evolves as a function of $\varepsilon_{e}$, but in a much weaker manner than for the previous case of $X_{\Sigma}=0.1$. This indicates that the main softening mechanism in this high-triaxiality situation $\left(X_{\Sigma}=1\right)$ is clearly the evolution of porosity which is found to lead to significant softening of the effective response of the porous material. Note that this void growth mechanism leads to the well-known 'high-triaxiality coalescence' of the voids, and failure consistent with the deep dimples observed in the micrographs from the experimental results of Barsoum and Faleskog (2007a). Also, it is clear that the dominance of the evolution of porosity will prevail at larger stress triaxialities $X_{\Sigma}>1$ not shown here (but see Danas and Ponte Castañeda (2009b)). 
a)

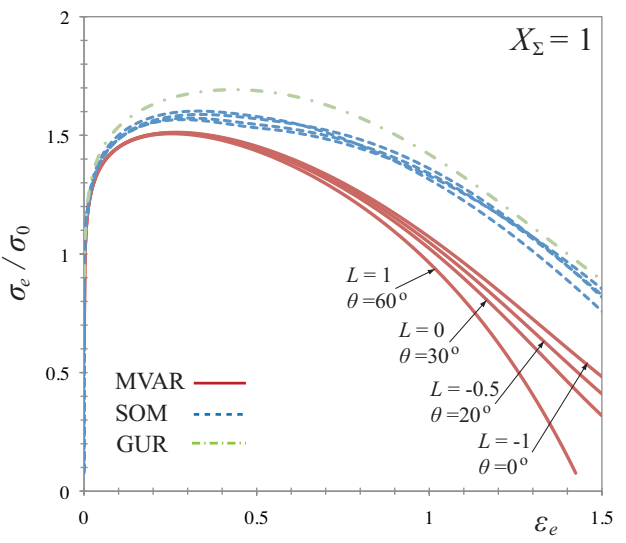

c)

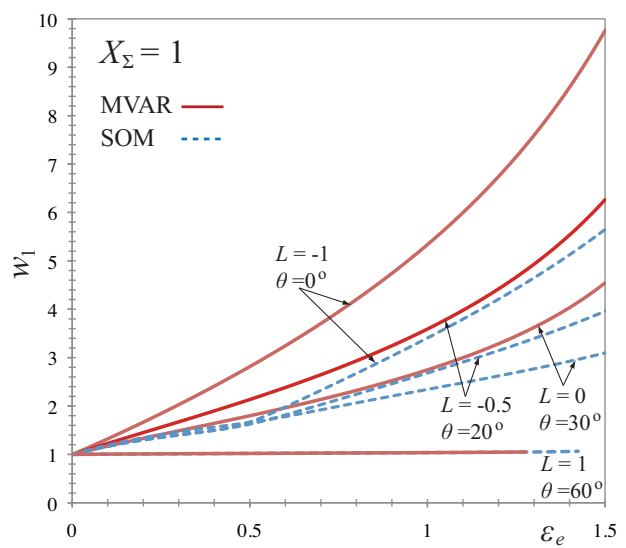

b)

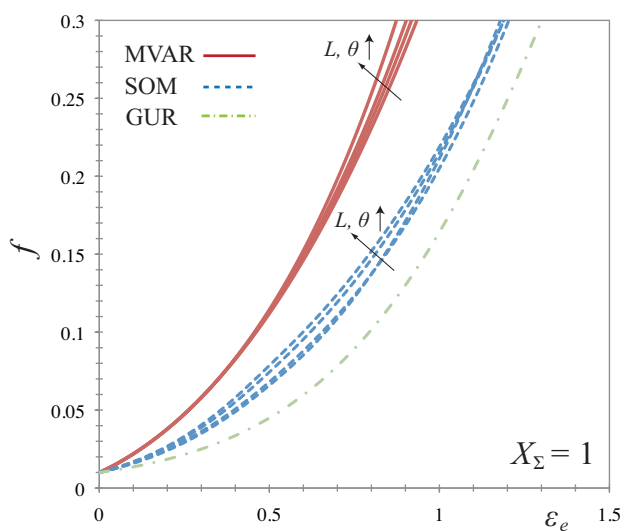

d)

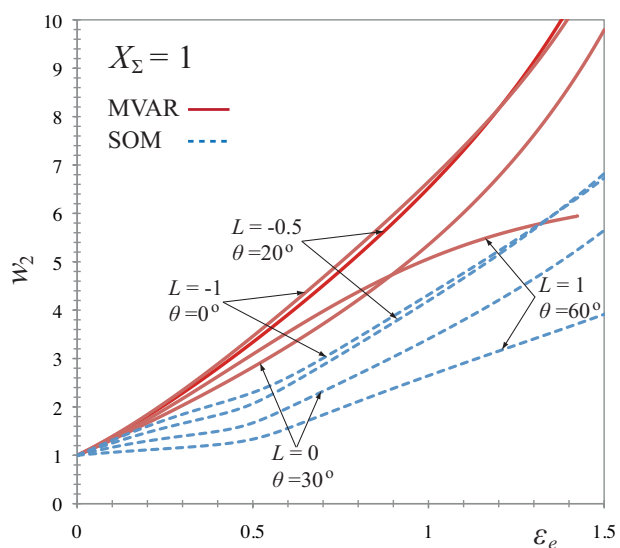

Figure 7: Plots of the MVAR, SOM and GUR estimates for (a) the equivalent stress $\sigma_{e}$, (b) the porosity $f$, and the aspect ratios (c) $w_{1}$ and (d) $w_{2}$ as a function of the equivalent strain $\varepsilon_{e}$, for a high value of the stress triaxiality $\left(X_{\Sigma}=1\right)$ and four values of the Lode parameter. The influence of the Lode parameter becomes negligible in this case since the response of the porous material is dominated by the significant evolution of porosity $f$. The strain hardening exponent is $N=0.1$ and the initial porosity $f_{0}=1 \%$.

\subsection{Remarks}

To summarize, the MVAR and the SOM predictions for the instantaneous material response are in good agreement for both isotropic and anisotropic microstructures. In particular, it has been observed that void shapes have significant effects on the instantaneous response of the porous material for a given porosity. We have shown that arbitrary ellipsoidal shapes lead (except in a small range of stress triaxialities) to a more compliant material response than the spheroidal shapes (prolate or oblate). Thus, ad-hoc approximations (see for instance Scheyvaerts et al. (2011)), where the void shape is assumed to remain spheroidal even for plane-strain loadings, require, first, the addition of several ad-hoc fitting parameters and, second, they could lead to inaccurate estimates for material failure since they tend to predict a stiffer material response.

In the context of finite deformations which lead to evolution of the microstructure, the MVAR and the SOM exhibit very similar qualitative behavior in both cases considered here (low triaxiality, $X_{\Sigma}=0.1$, and high triaxiality, $X_{\Sigma}=1$ ) although quantitatively we observe differences, albeit small ones. Nevertheless, 
it is important to note that neither of these two models have been confronted against direct experimental results (see for instance Boisot et al. (2011) for relevant work concerning a modification of the Gurson model) and hence the quantitative accuracy of any of the two remains an open question at this stage. In view of this, the MVAR model, being simpler than the SOM model, is numerically implemented in a user-material subroutine coupled with finite elements allowing to analyze qualitatively and quantitatively real geometries. Preliminary numerical results obtained with the MVAR and the Gurson model are discussed in the following section.

\section{Three dimensional simulations of a butterfly specimen}

Numerical simulations are carried out in the butterfly specimen sketched in Fig. 8. This specimen has originally been designed by Mohr and Henn (2007) and recently has been further optimized by Dunand and Mohr (2011). In the present study, we use the recently optimized specimen of Dunand and Mohr (2011), whereas the corresponding geometrical constants are summarized in Table 1. The present geometry allows for a wide range of stress and strain states in the middle section of the specimen by appropriate adjustment of the displacement ratio $u_{x} / u_{y}$ applied at the upper boundary of the specimen, as shown at the top of Fig. 8. The numerical calculations have been carried out in Abaqus (2009) using 6-noded triangular and 8-noded cubic linear, hybrid (with constant pressure) elements (C3D6H and C3D8H, respectively).
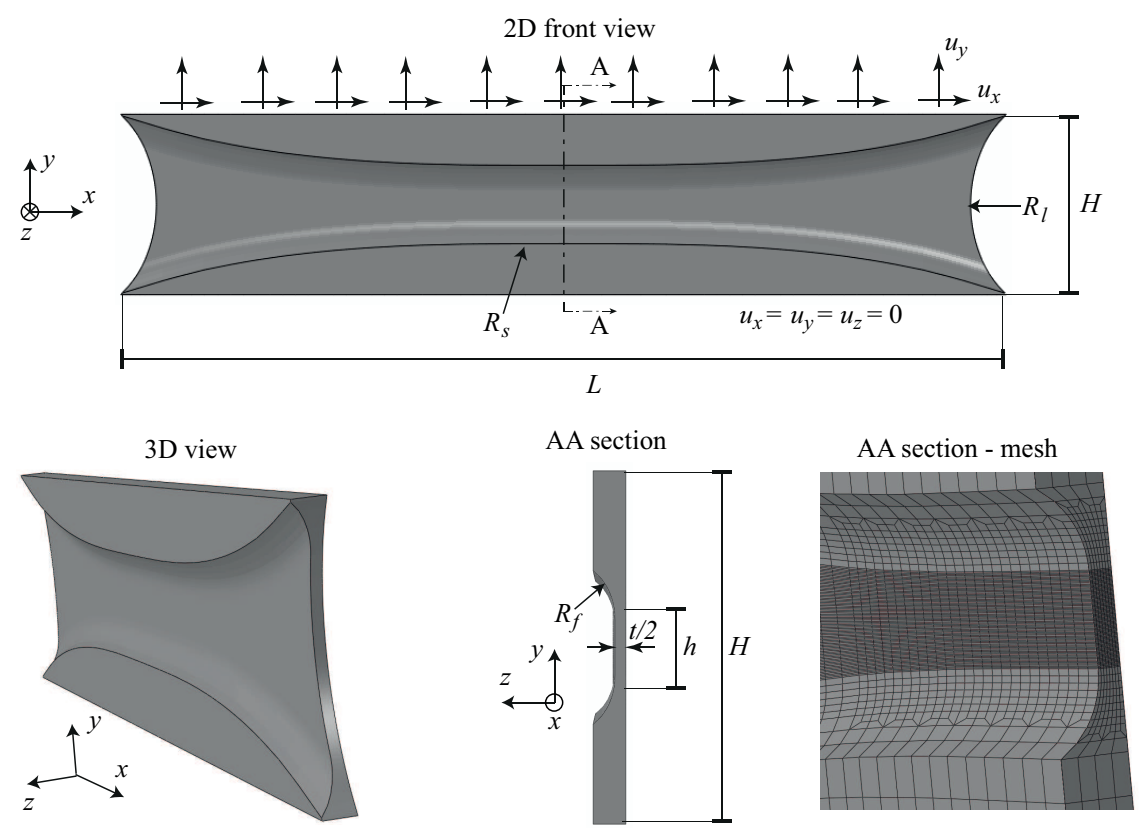

Figure 8: Three-dimensional butterfly geometry used in the simulations (courtesy of Matthieu Dunand and Prof. Dirk Mohr).

Table 1: Simulation Constants

\begin{tabular}{ccccccc|cccccccc}
\hline \multicolumn{1}{c}{ Geometry $(\mathrm{mm})$} & \multicolumn{1}{c}{ Material properties } \\
\hline \hline$H$ & $L$ & $h$ & $t$ & $R_{s}$ & $R_{l}$ & $R_{f}$ & $E / \sigma_{0}$ & $\nu$ & $\sigma_{0}$ & $N$ & $f$ & $w_{1}$ & $w_{2}$ \\
12.6 & 61.2 & 2 & 0.4 & $\sim 200$ & 10 & 2 & 300 & 0.3 & 1 & 10 & 0.01 & 1 & 1 \\
\hline
\end{tabular}

In our study, we consider two loading states. The first is a shear-dominated deformation load and is attained by fixing the ratio $u_{x}=10 u_{y}$ which leads to rather low stress triaxialities (at least before localization 
occurs) in the middle section of the specimen. Note that we do not choose to set $u_{y}=0$ with a non-zero $u_{x}$ in order to avoid difficulties with numerical convergence due to the very strong shear localization of the specimen in this case. The second load considered in the present study is a uniaxial tension load where $u_{y}>0$ (with $u_{x}=0$ ) which leads to higher stress triaxialities in the middle section of the specimen.

Then, we compare the results obtained by using the present modified variational model (MVAR) and the Gurson (1977) (GUR) model for the above stated loading conditions. The parameters used for the description of the porous material are summarized in Table 1 . The initial porosity is considered to be $f_{0}=1 \%$ while no porosity nucleation criteria are used during the calculation. In addition, it is noted that the pores are considered to be initially spherical in the MVAR model, such that $w_{1}=w_{2}=1$, whereas as the loading progresses the pores change in shape (become ellipsoidal) and orientation. On the other hand, in the Gurson model, the voids remain spherical during the entire deformation process.

\subsection{Shear-dominated loading}

Figure 9 shows MVAR and GUR estimates for the horizontal macroscopic force $F_{x}$ as a function of the macroscopic applied horizontal displacement $u_{x}$ in the case of the shear-dominated loading, i.e., $u_{x}=10 u_{y}$. The initial porosity for both models is $f_{0}=1 \%$ and the hardening exponent is $N=0.1$. We observe that up to a value $u_{x} \sim 3$, the response predicted by both models is very similar. However, following this value, the MVAR response exhibits a sharp drop in $F_{x}$, which is directly related to a very fast rate of localization of deformation (Rice, 1976) in a sharp zone in the gage section of the specimen, contrary to the GUR estimates which predict a much slower rate of localization in this section. The reason for this very sharp response of the MVAR model is related to the underlying porosity and void shape changes which lead to a more compliant response (locally) than the GUR model, as already discussed in the previous sections.

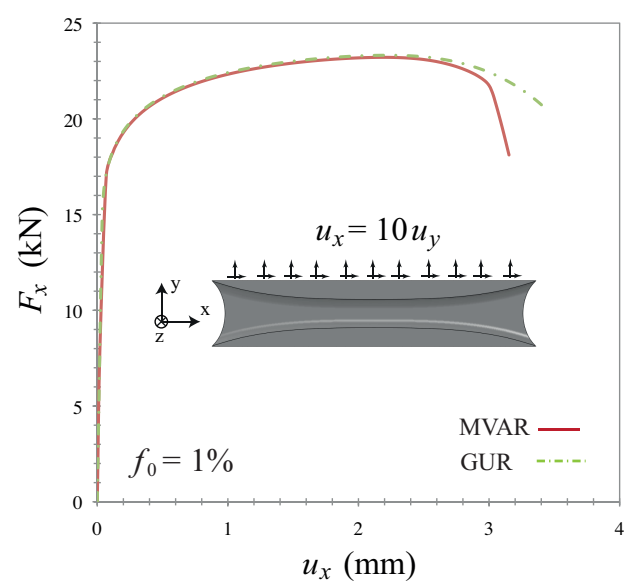

Figure 9: MVAR and GUR estimates for the horizontal macroscopic force $F_{x}$ as a function of the macroscopic applied horizontal displacement $u_{x}$. The loading is shear-dominated with $u_{x}=10 u_{y}$ at the upper surface of the specimen. The strain hardening exponent is $N=0.1$ and the initial porosity $f_{0}=1 \%$.

In order to examine the local response of the porous material, we choose a single element where porosity is maximum for both MVAR and GUR models ${ }^{3}$. As shown in the contours of Fig. 10, the maximum porosity for both the MVAR and the GUR model occurs in the upper right part of the gage section of the specimen and the zone of maximum porosity for both models is not parallel to the $x$-direction as already discussed in the experimental findings of Dunand and Mohr (2011). However, the MVAR predicts a higher value of porosity than the GUR model at the displacement level where the horizontal macroscopic force $F_{x}$ is maximum, respectively.

\footnotetext{
${ }^{3}$ Note that in general maximum porosity does not occur at the point of maximum accumulated plastic strain, since maximum $f$ depends only upon the hydrostatic part of the plastic strain tensor.
} 

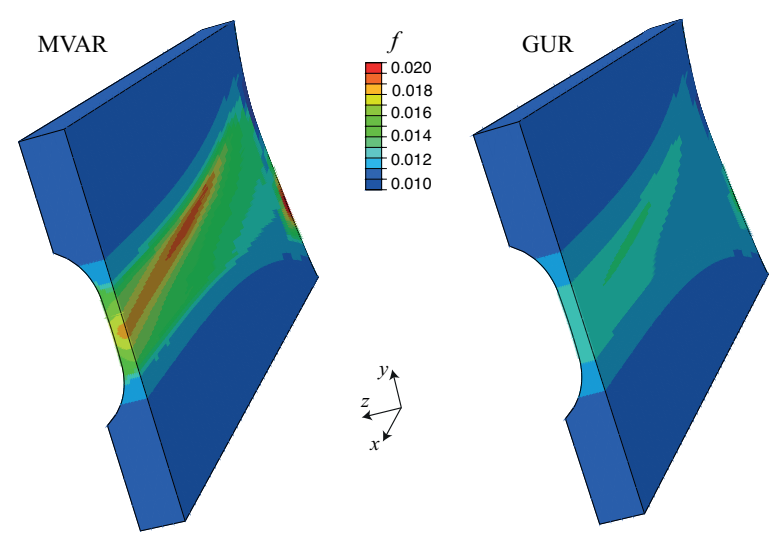

Figure 10: Porosity contours for the MVAR and the GUR models at the point where the horizontal macroscopic force $F_{x}$ is maximum, respectively.

a)

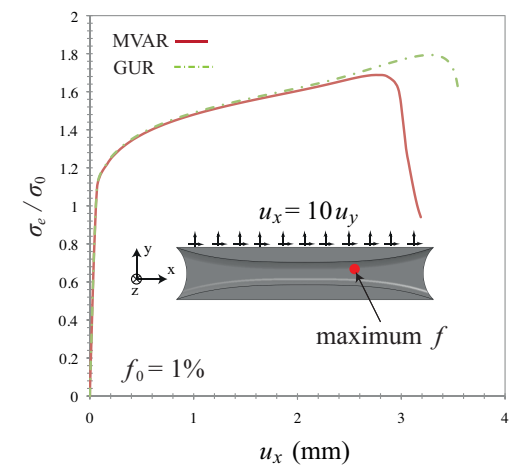

d)

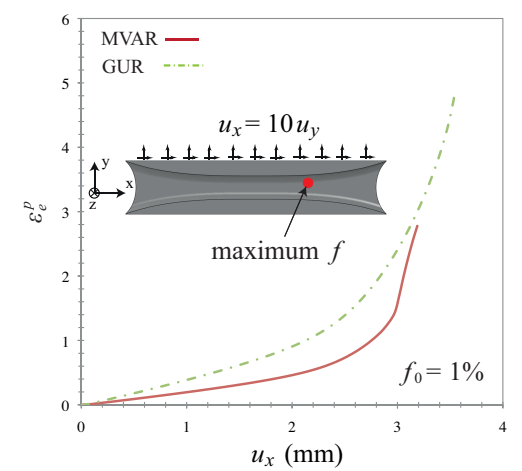

b)

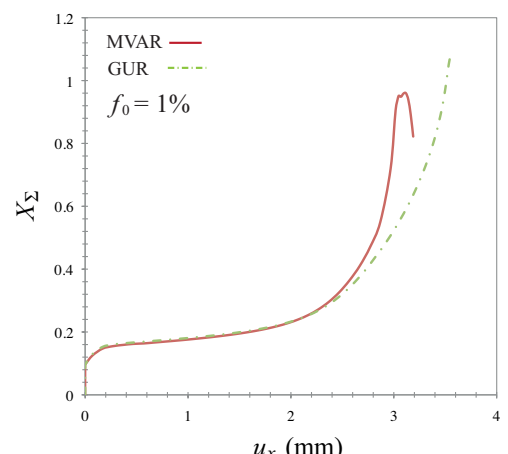

e)

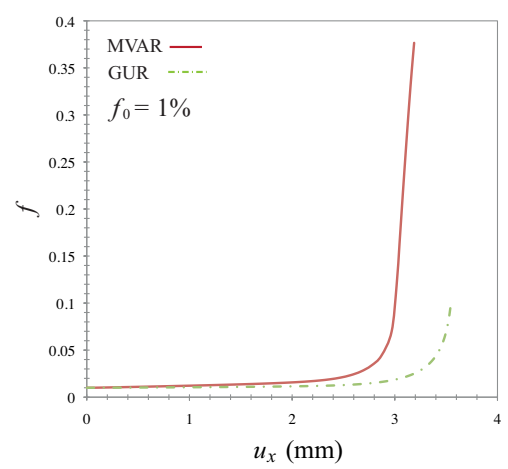

c)

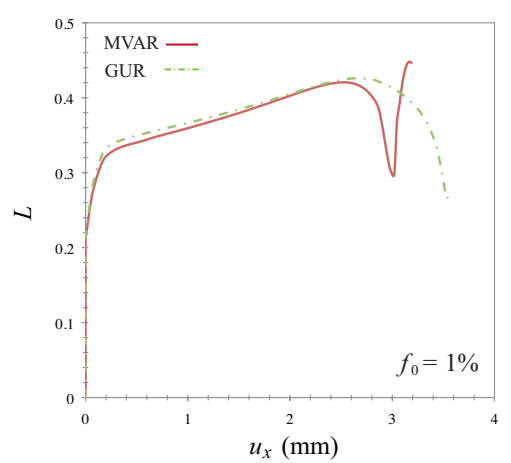

f)

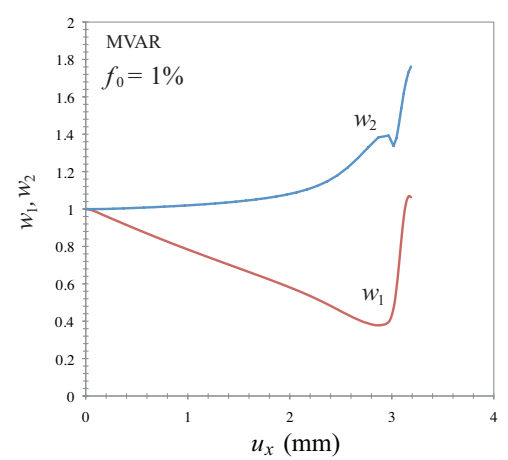

Figure 11: Plots of the MVAR and GUR estimates for (a) the equivalent stress $\sigma_{e}$, (b) the stress triaxiliaty $X_{\Sigma}$, (c) the Lode parameter $L,(\mathrm{~d})$ the effective plastic strain $\varepsilon_{e}^{p}$, (e) the porosity $f$, and (f) the aspect ratios $w_{1}$ and $w_{2}$ as a function of the macroscopic applied horizontal displacement $u_{x}$ at a single element where porosity is maximum (see red dot on the sketch of parts (a) and (d)). The loading is shear-dominated with $u_{x}=10 u_{y}$ at the upper surface of the specimen. The strain hardening exponent is $N=0.1$ and the initial porosity $f_{0}=1 \%$.

To investigate these differences between the MVAR and the GUR models, we plot the evolution of the 
microstructure at the centroid of a selected element which coincides with that maximum porosity point. Thus, Fig. 11 shows MVAR and GUR estimates for (a) the equivalent stress $\sigma_{e}$, (b) the stress triaxiality $X_{\Sigma}$, (c) the Lode parameter $L,(\mathrm{~d})$ the effective plastic strain $\varepsilon_{e}^{p}$, (e) the porosity $f$, and (f) the aspect ratios $w_{1}$ and $w_{2}$ as a function of the macroscopic applied horizontal displacement $u_{x}$. The main observation in the context of Fig. 11a is that at this local point, the MVAR exhibits a sharp stress drop and deformation localization at the zone of maximum porosity (see Fig. 10) much earlier than the GUR model. Similarly, the evolution of the stress triaxiality (Fig. 11b) and the Lode parameter (Fig. 11c) become very different for the two models analyzed here after the stage that the MVAR shows the sharp stress drop. In this connection, it should be pointed out that although the specimen has been designed to produce rather uniform distributions and low values of the stress triaxiality and the Lode parameter, we observe that after $u_{x} \sim 2.5$, both the MVAR and the GUR models predict a significant increase of the stress triaxiality. This is a direct consequence of the high value of porosity at this point that leads to localization of deformation and subsequent necking of the specimen in a thin zone. Thus, in the present example, even at initially low triaxiality loading conditions, we cannot prevent material necking that leads to an overall increase of the stress triaxialities at values of the order $\sim 1$. Deformation localization, in turn, leads to mesh dependence and thus has to be taken into account possibly by using gradient damage models (see for instance, (Feld-Payet et al., 2011)).

In Figs. 11d,e,f, we show the evolution of the underlying microstructure as predicted by the MVAR and the GUR models. Although, GUR model predicts a higher increase of the effective plastic strain $\varepsilon_{e}^{p}$ (Figs. 11d) than the MVAR model, the MVAR estimate for the corresponding porosity evolution (Figs. 11e) is much higher than that of GUR. This behavior can be explained by observing that the MVAR predicts a significant evolution of void shapes, i.e., of the aspect ratios $w_{1}$ and $w_{2}$ in Fig. 11f. In turn, this change in void shape leads to a strong porosity evolution and earlier deformation localization and failure (i.e., drop of the stress) of the material in that specific zone. Note that the rather fast increase of $w_{1}$ and $w_{2}$ in Fig. $11 \mathrm{f}$ at the later stages of the deformation process are a direct consequence of the fast increase of the stress triaxiality at this later stage. It is also useful to note that, for the shear dominated loading considered here, the aspect ratios in the MVAR evolve continuously during the entire deformation process. In contrast, porosity remains very low until values of $u_{x} \sim 2.5$ for the MVAR and $u_{x} \sim 3.2$ for the GUR, whereafter it exhibits a very fast increase leading to the fast drop of the stress observed in Fig. 11a.

\subsection{Uniaxial tension loading}

Figure 12 shows MVAR and GUR estimates for the vertical macroscopic force $F_{y}$ as a function of the macroscopic applied vertical displacement $u_{y}$ in the case of a uniaxial tension loading, i.e., $u_{x}=0$ and $u_{y}>0$. The initial porosity for both models is $f_{0}=1 \%$ and the hardening exponent is $N=0.1$. We observe that although, the MVAR model predicts a maximum load at lower values of the applied displacement $u_{y}$ than the GUR model, overall, both the MVAR and the GUR models exhibit a very similar response. Contrary to the shear-dominated loading discussed previously, the MVAR does not show a sharp drop in $F_{y}$ but a rather smooth material softening.

Nonetheless, the response is very different when we analyze a single element where porosity is maximum for both MVAR and GUR models. As shown in the contours of Fig. 13, the maximum porosity for both the MVAR and the GUR model occurs at the center of the gage section of the specimen and the zone of maximum porosity for both models is parallel to the $x$-direction as discussed in the the experimental findings of Dunand and Mohr (2011). Note however that the MVAR predicts a much higher value of porosity than the GUR model at the displacement level where the vertical macroscopic force $F_{y}$ is maximum, respectively.

To investigate these differences between the MVAR and the GUR models, we plot the evolution of the microstructure at the centroid of a selected element which coincides with that maximum porosity point. Thus, Fig. 14 shows MVAR and GUR estimates for (a) the equivalent stress $\sigma_{e}$, (b) the stress triaxiality $X_{\Sigma}$, (c) the Lode parameter $L,(\mathrm{~d})$ the effective plastic strain $\varepsilon_{e}^{p}$, (e) the porosity $f$, and (f) the aspect ratios $w_{1}$ and $w_{2}$ as a function of the macroscopic applied vertical displacement $u_{y}$. The main observation in the context of Fig. 14a is that the MVAR exhibits a maximum stress and subsequent deformation localization at the zone of maximum porosity (see Fig. 13) much earlier than the GUR model. The evolution of the stress triaxiality in Fig. 14b is identical for both the MVAR and the GUR models up to the point where the MVAR shows the sharp stress drop. Nonetheless, both the MVAR and the GUR model predict a significant 


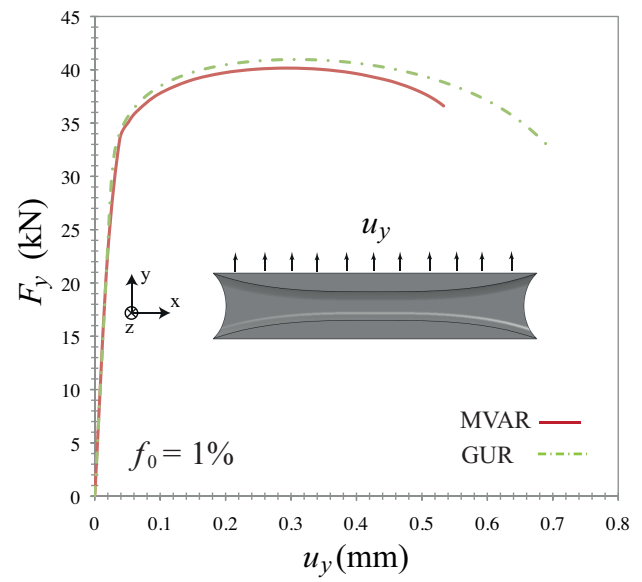

Figure 12: MVAR and GUR estimates for the vertical macroscopic force $F_{y}$ as a function of the macroscopic applied vertical displacement $u_{y}$. The loading is uniaxial tension along the $y$-direction with $u_{x}=0$ and $u_{y}>0$ at the upper surface of the specimen. The strain hardening exponent is $N=0.1$ and the initial porosity $f_{0}=1 \%$..
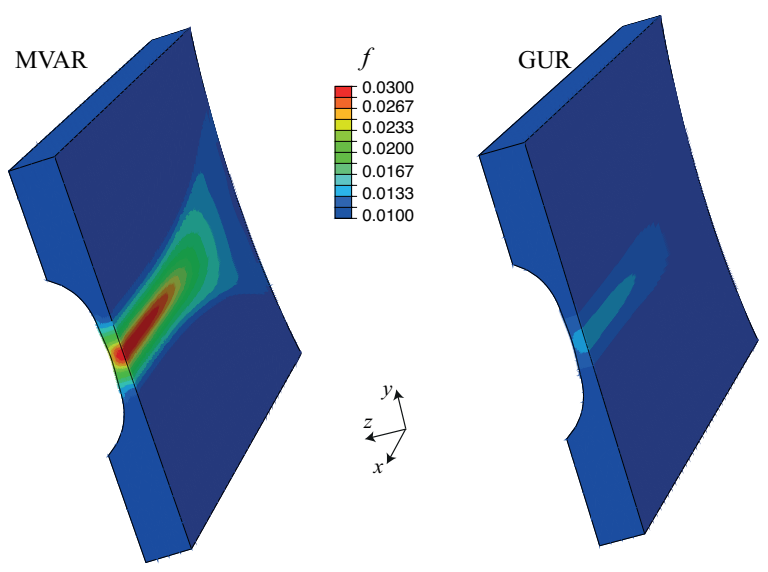

Figure 13: Porosity contours for the MVAR and the GUR models at a displacement level where the vertical macroscopic force $F_{y}$ is maximum, respectively.

increase of the stress triaxiality after the maximum stress point which can reach values in the excess of 1.2 . In Fig. 14c, we observe that both the MVAR and the GUR predict a very low value for the Lode parameter ( $L \sim 0.1$ for MVAR and $L \sim 0$ for GUR) until the maximum stress point where $L$ increases rapidly to values greater than 0.7 .

In Figs. 11d,e,f, we show the evolution of the underlying microstructure as predicted by the MVAR and the GUR models. Contrary to the previously discussed shear-dominated loading, in the case of uniaxial loading, the MVAR model predicts higher values for the effective plastic strain $\varepsilon_{e}^{p}$ than the GUR model, as shown in Fig. 11d. In particular, it is noted that even though the MVAR model leads to maximum stress and subsequent deformation localization at lower values of the macroscopic displacement $u_{y}$ than the GUR model, the corresponding plastic strains are much higher than those of the GUR model. In addition, porosity evolution (Fig. 11e) is much higher for the MVAR than the GUR model and this is directly attributed to the strong evolution of the void shapes as this is described by the very low values of the aspect ratios $w_{1}$ and $w_{2}$ in Fig. 11f. 
a)

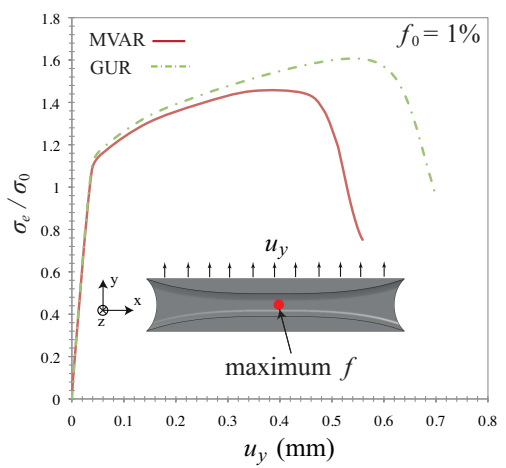

d)

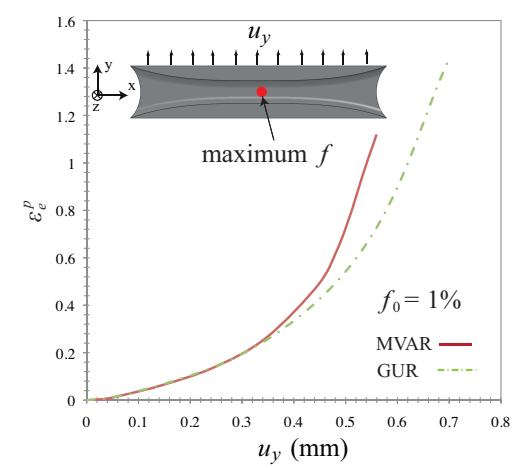

b)

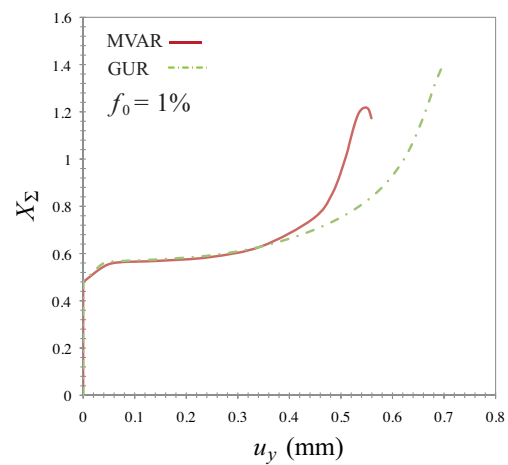

e)

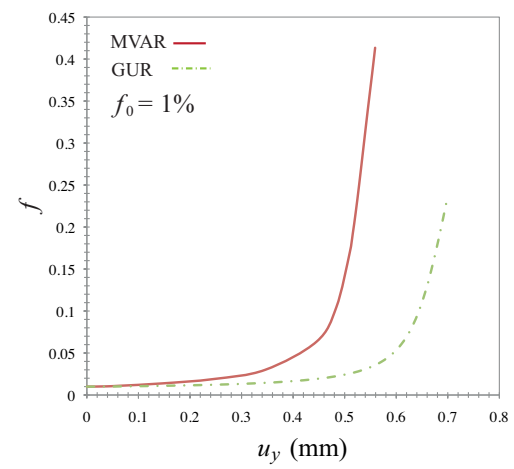

c)

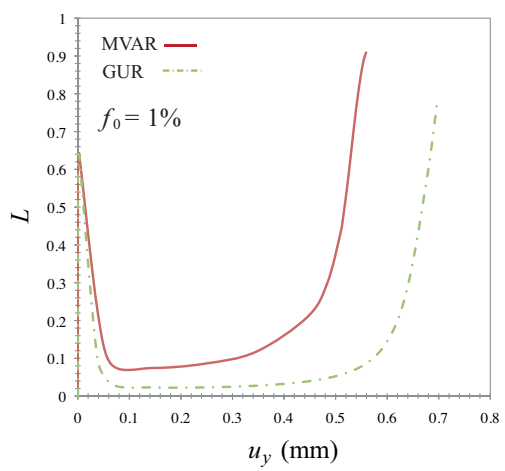

f)

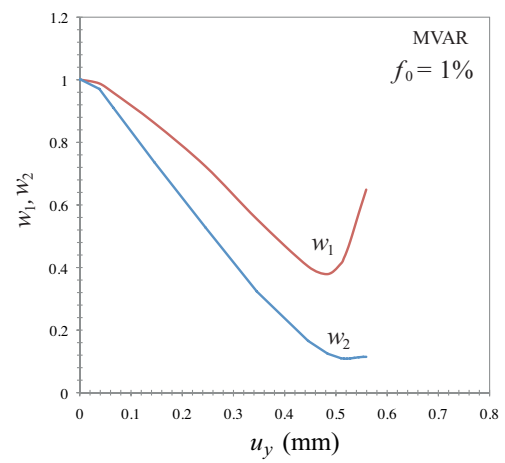

Figure 14: Plots of the MVAR and GUR estimates for (a) the equivalent stress $\sigma_{e}$, (b) the stress triaxiliaty $X_{\Sigma}$, (c) the Lode parameter $L,(\mathrm{~d})$ the effective plastic strain $\varepsilon_{e}^{p}$, (e) the porosity $f$, and (f) the aspect ratios $w_{1}$ and $w_{2}$ as a function of the macroscopic applied vertical displacement $u_{y}$ at a single element where porosity is maximum (see red dot on the sketch of parts (a) and (d)). The loading is uniaxial tension along the $y$-direction with $u_{x}=0$ and $u_{y}>0$ at the upper surface of the specimen. The strain hardening exponent is $N=0.1$ and the initial porosity $f_{0}=1 \%$.

\subsection{Remarks}

To summarize, simulations have been carried out for the butterfly geometry of Dunand and Mohr (2011) using the present modified variational model (MVAR) and the Gurson (1977) (GUR) model. It has been found that the presence of void shape effects, as is the case in the new MVAR model, leads to maximum load, deformation localization and failure of the material at lower values of the macroscopic applied displacement when compared with the GUR model which contains no void shape effects. However, the corresponding local plastic strains at failure predicted by the MVAR model are not necessarily lower than the those of the GUR model. It has been found, for example, that for the uniaxial loading case the local plastic strains at the position of maximum porosity are much higher for the MVAR model than the GUR model. Moreover, for both the MVAR and the GUR model, maximum stress occurs locally due to the underlying microstructure evolution (this is not the case for the standard $J_{2}$ plasticity theories which cannot exhibit locally a maximum stress load). The microstructure evolution, in turn, provides information about the potential local failure of the material (i.e., position of maximum porosity and void shape elongation at zone of high shears) which precedes always the macroscopic failure of the structure. Note further that after the maximum stress load occurs both stress triaxiality and Lode parameter increase significantly and in a different manner for the MVAR and the GUR models. Consequently, this non-proportional response has to be taken into account in the construction of failure maps (see for instance Bao and Wierzbicki (2004) and Barsoum and Faleskog 
(2007a)) as functions of the stress triaxiality and the effective (accumulated) plastic strain. In addition, the fact that the MVAR and the GUR models predict rather different values for the macroscopic displacement where maximum load occurs, implies that use of different models to analyze experimental results could lead to very different quantitative observations even when the initial porosity is as small as $1 \%$.

Finally, it is clear from the previous simulations that special care needs to be considered after maximum load occurs locally in some section of the specimen. It is well known that strong softening of the material leads to localized deformation and consequently to mesh size dependence. In this case of excessive deformation localization, the present micromechanical models have to be augmented with appropriate non-local criteria (see for instance (Feld-Payet et al., 2011)) in order to assess material failure and fracture after the maximum stress point in real experimental studies. Such work is in progress.

\section{Acknowledgments}

The authors would like to thank Matthieu Dunand and Professor Dirk Mohr of the Ecole Polytechnique for the butterfly specimen geometry and mesh, which allowed for the three-dimensional simulations in the last section of this work.

Availability of computer programs: Copies of the computer code (UMAT) or independent subroutines for the calculations of the Eshelby tensors will be supplied upon request. Please address inquiries to Dr. Kostas Danas at the e-mail address kdanas@lms.polytechnique.fr.

\section{References}

ABAQUS/Standard Version 6.9,2009. Users Manual, Dassault Systems, Simulia Corp., Providence, RI, USA.

Aravas, N., 1992. Finite elastoplastic transformations of transversely isotropic metals. Int. J. Solids Struct. $29,2137-2157$.

Aravas, N., Ponte Castañeda, P., 2004. Numerical methods for porous metals with deformation-induced anisotropy. Comput. Methods Appl. Mech. Engng. 193, 3767-3805.

Bao, Y., Wierzbicki, T., 2004. On fracture locus in the equivalent strain and stress triaxiality space. Int. J. Mech. Sci. 46 (81), $81-98$.

Barsoum, I., Faleskog, J. 2007a. Rupture mechanisms in combined tension and shearExperiments. Int. J. Solids Struct., 44, 1768-1786.

Benzerga, A. A., 2002. Micromechanics of coalescence in ductile fracture. J. Mech. Phys. Solids 50, $1331-1362$.

Benzerga, A. A., Besson, J., Pineau, A, 2004a. Anisotropic ductile fracture: Part I: experiments Acta Materialia, 52, 4623-4638

Benzerga, A. A., Besson, J., Pineau, A, 2004b. Anisotropic ductile fracture: Part II: theory Acta Materialia, 52, 4639-4650

Benzerga, A. A., Leblond, J.-B., 2010. Ductile Fracture by Void Growth to Coalescence. Adv. Appl. Mech. 44, $170-297$.

Boisot, G., Laiarinandrasana, L., Besson, J., Fondc, C., Hochstetterb, G.,2011. Experimental investigations and modeling of volume change induced by void growth in polyamide 11 Int. J. Solids Struct., 48, 2642-2654.

Budiansky, B., Hutchinson, J. W., Slutsky, S., 1982. Void growth and collapse in viscous solids. Mechanics of Solids, The Rodney Hill 60th aniversary Volume, Hopkins, H. G. and Sewell, M. J., eds., Pergamon Press Oxford 13-45.

Chu, C.C., Needleman, A., 1980. Void nucleation effects in biaxially stretched sheets. J. Engrg. Mat. Tech. 102, $249-256$.

Dafalias, Y., F., 1985. The plastic spin. J. Appl. Mech. 52, 865-871.

Danas, K., 2008. Homogenization-based constitutive models for viscoplastic porous media with evolving microstructure. Ph.D. thesis, LMS, École Polytechnique: http://www.polymedia.polytechnique.fr/Center.cfm?Table=These.

Danas, K., Idiart, M.I., Ponte Castañeda, P., 2008a. Homogenization-based constitutive model for two-dimensional viscoplastic porous media. C. R. Mecanique 336, 79-90.

Danas, K., Idiart, M.I., Ponte Castañeda, P., 2008b. Homogenization-based constitutive model for isotropic viscoplastic porous media. Int. J. Solids Struct. 45, 3392-3409.

Danas, K., Ponte Castañeda, P., 2009a. A finite-strain model for anisotropic viscoplastic porous media: I - Theory. Eur. J. Mech. A/Solids 28, 387-401.

Danas, K., Ponte Castañeda, P., 2009b. A finite-strain model for anisotropic viscoplastic porous media: II - Applications. Eur. J. Mech. A/Solids 28, 402-416.

Danas, K., Ponte Castañeda, P.. Influence of the Lode parameter and the stress triaxiality on the failure of elasto-plastic porous materials. under review.

Dunand, M., Mohr, D., 2011. Optimized butterfly specimen for the fracture testing of sheet materials under combined normal and shear loading. Eng. Fract. Mech. in press, doi:10.1016/j.engfracmech.2011.08.008.

Dunand, M., Mohr, D., 2010. Hybrid experimental-numerical analysis of basic ductile fracture experiments for sheet metals. Int. J. Solids Struct. 47, 1130-1143. 
Eshelby, J.D., 1957. The determination of the elastic field of an ellipsoidal inclusion and related problems. Proc. R. Soc. Lond. A $241,376-396$.

Feld-Payet, S., Besson, J., Feyel, F., 2011. Finite Element Analysis of Damage in Ductile Structures Using a Nonlocal Model Combined with a Three-field Formulation. Int. J. Damage Mechanics 20, 655-680.

Flandi, L., Leblond, J.-B., 2005a. A new model for porous nonlinear viscous solids incorporating void shape effects - I: Theory, Eur. J. Mech. A/Solids 24, 537-551.

Fleck, N. A., Hutchinson, J. W., 1986. Void growth in shear. Proc. R. Soc. Lond. A 407, 435-458.

Gărăjeu, M., Michel, J.-C., Suquet, P., 2000. A micromechanical approach of damage in viscoplastic materials by evolution in size, shape and distribution of voids. Comp. Methods Appl. Mech. Engrg. 183, 223-246.

Garrison Jr., W.M.., Moody, N.R.,1987. Ductile fracture. J. Phys. Chem. Solids 48, 1035-1074.

Ghahremaninezhad, A., Ravi-Chandar, K., 2011. Ductile failure in polycrystalline OFHC copper Int. J. Solids Struct., In Press, Corrected Proof, doi:10.1016/j.ijsolstr.2011.07.001.

Gologanu, M., Leblond, J.-B., Devaux, J., 1993. Approximate models for ductile metals containing non-spherical voids - case of axisymmetric prolate ellipsoidal cavities. J. Mech. Phys. Solids 41, 1723-1754.

Gologanu, M., Leblond, J.-B., Devaux, J., 1994. Approximate models for ductile metals containing non-spherical voids - case of axisymmetric oblate ellipsoidal cavities. ASME J. Engrg. Materials Technol. 116, 290-297.

Gologanu, M., Leblond, J.-B., Devaux, J., 1997. Recent extensions of Gurson's model for porous ductile metals. Suquet, P. (Ed.), Continuum micromechanics. In: CISM lectures series. Springer, New York, 61-130.

Gurson, A.L., 1977. Continuum theory of ductile rupture by void nucleation and growth. J. Engng. Mater. Technol. 99, 2-15.

Hashin, Z., 1962. The elastic moduli of heterogeneous materials. J. Appl. Mech, 143-150.

Hill, R. 1978. Aspects of invariance in solids mechanics. Advances in Applied Mechanics 18, (ed.C-S Yih), Academic Press, New York, 1-75.

Idiart, M.I., Moulinec, H, Castañeda, P.P., Suquet, P, 2006. Macroscopic behavior and field fluctuations in viscoplastic composites: Second-order estimates versus full-field simulations J. Mech. Phys. Solids 46, 1029-1063.

Idiart, M.I., 2008a. Modeling the macroscopic behavior of two-phase nonlinear composites by infinite-rank laminates Journal J. Mech. Phys. Solids 56, 2599-2617.

Idiart, M.I., 2008b. The macroscopic behavior of power-law and ideally plastic materials with elliptical distribution of porosity. Mec. Res. Commun., 35, 583-588.

Johnson, G.R., Cook, W.H., 1985. Fracture characteristics of three metals subjected to various strains, strain rates, temperatures and pressures. Engrg. Fracture Mech. 21 (1), 31-48.

Kailasam, M., Aravas, N., Ponte Castañeda, P. 2000. Porous metals with developing anisotropy: Constitutive models, computational issues and applications to deformation processing. Computer Modeling in Engineering and Sciences 1, $105-118$.

Kailasam, M., Ponte Castañeda, P., 1997. The evolution of anisotropy in porous materials and its implications for shear localization. IUTAM Symposium on Mechanics of Granular and Porous Materials, N.A. Fleck and A.C.F. Cocks, Eds., Kluwer Academic Publishers, 365-376.

Kailasam, M., Ponte Castañeda, P., 1998. A general constitutive theory for linear and nonlinear particulate media with microstructure evolution. J. Mech. Phys. Solids 46, 427-465.

Kailasam, M., Ponte Castañeda, P., and Willis, J. R., 1997a. The effect of particle size, shape, distribution and their evolution on the constitutive response of nonlinearly viscous composites. I. Theory. Phil. Trans. R. Soc. Lond. A 355, $1835-1852$.

Kailasam, M., Ponte Castañeda, P., and Willis, J. R., 1997b. The effect of particle size, shape, distribution and their evolution on the constitutive response of nonlinearly viscous composites. II. Examples. Phil. Trans. R. Soc. Lond. A 355, $1853-1872$.

Leblond, J.-B., Gologanu, M., 2008. External estimate of the yield surface of an arbitrary ellipsoid containing a confocal void. C. R. Mécanique, 336, 813-819

Leblond, J.-B., Perrin, G., Suquet, P., 1994. Exact results and approximate models for porous viscoplastic solids. Int. J. Plasticity 10, 213-235.

Michel, J.-C., Suquet, P., 1992. The constitutive law of nonlinear viscous and porous materials. J. Mech. Phys. Solids 40,783 -812 .

Mohr, D., Ebnoether, F., 2009. Plasticity and fracture of martensitic boron steel under plane stress conditions. Int. J. Solids Struct. 46, 3535-3547.

Mohr, D., Henn, S., 2007. Calibration of stress-triaxiality dependent crack formation criteria: a new hybrid experimentalnumerical method. Exp. Mech, 47, 805-820.

V. Monchiet, E. Charkaluk, D. Kondo, 2007. An improvement of Gurson-type models of porous materials by using Eshelby-like trial velocity fields, C. R. Mcanique, 335, 32-41.

Nahshon, K., Hutchinson, J. W., 2008. Modification of the Gurson model for shear failure. Eur. J. Mechanics A/Solids 27, $1-17$.

Needleman, A., Rice, J.R., 1978. Limits to ductility set by plastic flow localization. In: Koistinen, D.P., et al. (Eds.), Mechanics of Sheet Metal Forming. Plenum Publishing, 237-267.

Ponte Castañeda, P., 1991a. The effective mechanical properties of nonlinear isotropic composites. J. Mech. Phys. Solids 39, $45-71$.

Ponte Castañeda, P., 1991b. Effective properties in power-law creep. In Mechanics of Creep Brittle Materials 2, edited by A.C.F. Cocks and A.R.S. Ponter, 218-229. London: Elsevier, 1991.

Ponte Castañeda, P., 2002a. Second-order homogenization estimates for nonlinear composites incorporating field fluctuations. I. Theory. J. Mech. Phys. Solids 50, 737-757.

Ponte Castañeda, P., 2002b. Second-order homogenization estimates for nonlinear composites incorporating field fluctuations. II. Applications. J. Mech. Phys. Solids 50, 759-782. 
Ponte Castañeda, P., Willis, J.R., 1995. The effect of spatial distribution on the effective behavior of composite materials and cracked media. J. Mech. Phys. Solids 43, 1919-1951.

Ponte Castañeda, P., Zaidman, M., 1994. Constitutive models for porous materials with evolving microstructure. J. Mech. Phys. Solids 42, 1459-1497.

Rice, J.R., Tracey, D.M., 1969. On the ductile enlargement of voids in triaxial fields. J. Mech. Phys. Solids 17, $201-217$.

Rice, J. R. (1976). The localization of plastic deformation. Proceedings of the $14^{\text {th }}$ International Congress of Theoretical and Applied Mechanics, W. T. Koiter, ed., North-Holland Publishing Company, 207-220.

Scheyvaerts, F., Onck, P.R., Tekogolu, C., Pardoen, T., 2011. The growth and coalescence of ellipsoidal voids in plane strain under combined shear and tension. J. Mech. Phys. Solids 59, 373-397.

Suquet, P., 1993. Overall potentials and extremal surfaces of power law or ideally plastic materials. J. Mech. Phys. Solids 41, 981-1002.

Suquet, P., 1995. Overall properties of nonlinear composites: a modified secant moduli theory and its link with Ponte Castañeda's nonlinear variational procedure. C. R. Acad. Sci. Paris II 320, 563-571.

Tvergaard, V., 1981. Influence of voids on shear band instabilities under plane strain conditions. Int. J. Fracture $17,389-407$.

Tvergaard, V., 1990. Material failure by void growth. Adv. Appl. Mech. 27, 83-151.

Tvergaard, V., 2009. Behaviour of voids in a shear field. Int. J. Fracture 158, 41-49.

Vincent P-G., Monerie Y., 2008. Yield criterion for a rigid-ideally plastic material with randomly oriented cracks C. R. Mecanique, 336, 297-303.

Willis, J.R., 1978. Variational principles and bounds for the overall properties of composites. Continuum Models and Discrete Systems 2, (ed. J. Provan), 185-212.

Willis, J.R., 1981. Variational and related methods for the overall properties of composites. Adv. Appl. Mech. 21, 1-78.

Willis, J.R., 1991. On methods for bounding the overall properties of nonlinear composites. J. Mech. Phys. Solids 39, 73-86. 